\title{
A UKST survey of blue objects towards the Galactic centre - seven additional fields
}

\author{
P. L. Dufton ${ }^{1}$, S. J. Smartt ${ }^{2}$, and N. C. Hambly ${ }^{3}$ \\ 1 The Department of Pure and Applied Physics, The Queen's University of Belfast, Belfast BT7 1NN, N. Ireland, \\ UK \\ 2 Institute for Astronomy, University of Cambridge, Madingley Road, Cambridge CB3 0BA, UK \\ 3 Institute for Astronomy, University of Edinburgh, Blackford Hill, Edinburgh EH9 3HJ, Scotland, UK
}

Received 2 April 2001 / Accepted 27 April 2001

\begin{abstract}
We are searching for early-type stars towards the Galactic centre which are potentially young objects situated within the inner few kiloparsecs of the disk. Photographic photometry from the UK Schmidt Telescope has been used to identify the bluest candidates in nineteen Schmidt fields (centred close to the Galactic centre). We have previously obtained FLAIR low dispersion spectroscopy for three of these fields to estimate spectral types and here we present spectroscopy for an additional seven fields. Combining the results for all ten fields, 56 stars were initially classified as early-B type. Estimates of the equivalent widths of their Balmer and He I lines have been used to estimate atmospheric parameters and 32 targets have effective temperatures greater than or equal to $17000 \mathrm{~K}$ (corresponding to a spectral type of B3 or earlier). The spectra of seven of these targets also have absorption lines due to O II and Si III and can be reliably classified as early-B type. Additionally 78 stars have estimated effective temperatures between 11000 and $16000 \mathrm{~K}$ with a further a further 50 objects identified as late-B (or early-A) type. All but two of the early B-type candidates have magnitudes in the range $12.0 \leq V \leq 16.0$, and our best estimates of their distance suggest that they could be close to (i.e. $R_{\mathrm{g}}<3 \mathrm{kpc}$ ), or even beyond the Galactic centre.
\end{abstract}

Key words. stars: early-type - stars: fundamental parameters - stars: imaging - galaxy: center

\section{Introduction}

We are undertaking a survey to identify young early-type stars towards the centre of our Galaxy. The rationale and methodology were discussed by Dufton et al. (1999 - hereafter DSH), where results for three Schmidt fields were also presented. Early-type stars play an important role in the evolutionary history of galaxies (see, for example, the proceedings of the second Boulder-Munich workshop - Howarth 1998). Recent investigations include our Galaxy (Gummersbach et al. 1998; Rolleston et al. 2000), the Magellanic System (Korn et al. 2000; Rolleston et al. 1999), M31 (Smartt et al. 2001a) and M33 (Monteverde et al. 1997), with lower resolution observations possible for even more distant galaxies (e.g. Bresolin et al. 2001). Their short evolutionary lifetimes (typically 10 million years) ensure that early-type stars provide information on the "current" status of the host galaxy.

Stellar investigations of the chemical composition of the Galactic disk have concentrated mainly on the anticentre direction (Smartt \& Rolleston 1997; Gummersbach et al. 1998; Rolleston et al. 2000) and have provided reliable stellar estimate of large scale abundance gradients

Send offprint requests to: P. L. Dufton,

e-mail: p.dufton@qub.ac.uk for a relatively wide range of elements. Studies towards the Galactic centre have been hampered by the large extinction at optical wavelengths. However, preliminary investigations of stars with Galactic latitudes $2^{\circ} \leq|b| \leq 10^{\circ}$ have shown that it is possible to map both the chemical composition and stellar populations towards and beyond the Galactic centre (Smartt et al. 2001b; Ryans et al. 1997; Venn et al. 1998).

In order to further these studies, it is important to identify more potential targets and also go to fainter magnitudes than is available from published catalogues (Reed 1993; Reed \& Beatty 1995). This requires a wide-field survey technique employing both multi-colour photometry and preliminary low-resolution spectroscopy for spectraltype classification. The UK Schmidt telescope provides appropriate instrumentation to fulfill our needs. As discussed in DSH, suitable photographic material for a mosaic of 19 Schmidt fields (yielding a total survey area of 475 square degrees) has already been obtained, and we have measured a subset of 14 of these fields, to produce lists of candidate blue stellar objects. For three fields, low dispersion multi-object spectroscopy of the blue targets were obtained by DSH and here were present spectroscopy for a further seven fields. 
Table 1. The 10 Galactic Centre Survey fields for which spectroscopy has been obtained. Spectroscopy for the fields 391, 454 and 456 has been discussed by DSH.

\begin{tabular}{cccccc}
\hline $\begin{array}{c}\text { Field } \\
\text { no. }\end{array}$ & RA & \multicolumn{2}{c}{ DEC } & \multicolumn{2}{c}{ Exposure times } \\
(J2000.0) & Blue & (hours) & Red \\
\hline 279 & 174741 & -4501 & 3.5 & 2.5 \\
333 & 172329 & -4003 & 4.5 & 1.5 \\
391 & 165118 & -3505 & 4.0 & - \\
393 & 173920 & -3502 & 4.0 & 1.5 \\
454 & 171812 & -3003 & 4.0 & - \\
456 & 180412 & -3000 & 4.0 & 1.0 \\
520 & 173905 & -2502 & 3.5 & 1.0 \\
522 & 182305 & -2458 & 3.5 & 1.5 \\
589 & 175358 & -2001 & 3.0 & 1.0 \\
591 & 183558 & -1957 & 4.0 & 1.5 \\
\hline
\end{tabular}

${ }^{1}$ Standard United Kingdom Schmidt Telescope field numbers are defined in Tritton (1983); see also the UKSTU online catalogue at http://www.roe.ac.uk/

\section{Observational data}

\subsection{Photographic photometry}

The photographic photometry and its reduction has been discussed in detail by DSH and Fig. 1, taken from their paper, shows the Galactic centre survey region, and the 19 fields. These are labeled with standard UK Schmidt field numbers - see, for example, Tritton (1983). Fields were surveyed using existing short exposure $V$ plates from the plate library along with new $U$ films obtained specially for this work.

The photographs were scanned using the precision microdensitometer SuperCOSMOS (see, for example, Hambly et al. 1998) and stellar candidates identified and matched as discussed in DSH. An approximate calibration of the $V$-band instrumental magnitudes was obtained by fitting instrumental magnitudes versus $V$ photometry from the Tycho stars in each field. No independent data were available for calibration of the $U$-band photographic instrumental magnitudes; hence in order to make the blue object selection in each field, we used the $u-v$ colour index. Note that as discussed in DSH, we corrected this index to allow for zero-point offsets which are functions of magnitude. A typical pseudo colour-magnitude scatter plot is shown in Fig. 2 of DSH. This procedure then allowed us to select the bluest stars in each field in the range $11.5<V<16$ regardless of magnitude and in a consistent manner from field to field.

The relative accuracy of these colours was estimated by DSH to be at best $\sim 0.2^{\mathrm{m}}$. Also because the fields are very crowded, the de-blending algorithm will discard many overlapping images. However, for identifying early-type stellar candidates in the region of the Galactic centre, completeness is not a major consideration. The photometric data are available from the authors on request for the 7 fields which are discussed here, together with the 3 field previously discussed by DSH.

\subsection{Low resolution spectroscopy}

Low resolution spectroscopy was undertaken using the FLAIR instrument on the UK Schmidt Telescope at Siding Springs with seven nights being allocated in June 1999. Conditions were good throughout the run and one field was observed in both the red and blue spectral regions each night. The procedures adopted were similar to those discussed by DSH and only brief details are given here.

The FLAIR system with the $100 \mu \mathrm{m}$ diameter fibres allows the simultaneous observation of up to 90 objects (from which target and sky fibres need to be allocated) subject to limitations imposed by the finite size of the fibre ferrules and prisms. Typically between 30 and 50 fibres were positioned on stellar targets with typically 10 sky positions. The prioritisation of targets was primarily based on their colours, with the very blue targets only being excluded if proximity to another star made this necessary. Spectra were obtained covering the wavelength regions from approximately 3860 to $4640 \AA$ using the 1200B grating with a resolution (full-width-half-maximum) of approximately $2.7 \AA$ and from approximately 5400 to $6900 \AA$ at a resolution of approximately $5.9 \AA$ using the 600R grating. Further details of the FLAIR instrument can be found in Parker \& Watson (1994).

Preliminary data reduction was undertaken using the AAO's FLAIR package within the IRAF environment (Drinkwater \& Holman 1996) and followed the procedures discussed in DSH. The IRAF package dofibers (Valdes 1992) was used to extract the 1D spectra from each image, correct for the fibre-to-fibre variation in throughput (from the flat-field response frames), sky subtract, and then wavelength calibrate the final product.

The bluest stellar candidates, including all those for which spectroscopy was obtained, are summarised for each field in Tables 2 to 8 . These list the coordinates, magnitudes and colours and where available spectral types inferred from the low resolution spectroscopy. The spectra are available from the authors on request.

\section{Analysis}

\subsection{Spectral types}

The reduced spectra were input into the STARLINK package, DIPSO (Howarth et al. 1993) for further analysis. Initially the aim was to estimate spectral types for all the spectra which had a sufficient signal. For the blue spectra, the classification scheme is similar to that used by DSH, but for the red spectra, it has been significantly extended. Indeed DSH considered the red and blue spectra together when estimating spectral types. The different approach adopted here reflects the higher quality and more extensive nature of the red spectra than those that were available to DSH. 
Table 2. Blue stellar candidates for Field 279 .

\begin{tabular}{|c|c|c|c|c|c|c|c|c|}
\hline Star & RA (2000) & Dec & $V$ & $(u-v)$ & $C_{\mathrm{b}}$ & $\overline{C_{\mathrm{r}}}$ & Blue spectra & Red spectra \\
\hline 1 & 17380.47 & -45214.0 & 15.84 & -1.08 & 1400 & 100 & He rich & PN? \\
\hline 2 & 174217.14 & $-46 \quad 16 \quad 41.1$ & 14.78 & -0.80 & 910 & 0 & early-B & - \\
\hline 3 & 17490.03 & -465626.8 & 10.03 & -0.78 & & & & \\
\hline 4 & 17467.90 & -462323.5 & 15.42 & -0.76 & 630 & 0 & early-B & - \\
\hline 5 & 174740.69 & -42387.6 & 14.92 & -0.76 & 1100 & 1200 & early-B & $\mathrm{AB} ?$ \\
\hline 6 & 174855.26 & -43118.4 & 10.64 & -0.63 & & & & \\
\hline 7 & 173948.14 & -46853.9 & 14.43 & -0.62 & 1700 & 630 & early-B & - \\
\hline 8 & 18133.90 & -453156.4 & 12.37 & -0.62 & 1900 & 2500 & early-B & early-B \\
\hline 9 & 174314.69 & -45232.8 & 13.71 & -0.59 & 4500 & 2000 & early-B & late? \\
\hline 10 & 174242.80 & -441017.6 & 15.88 & -0.58 & 1800 & 1400 & late-B & - \\
\hline 11 & 175633.72 & -43319.6 & 13.82 & -0.58 & 80 & 1300 & $\mathrm{PN}$ & $\mathrm{PN}$ \\
\hline 12 & 174234.04 & -465848.8 & 11.18 & -0.57 & 14000 & 12000 & late & late \\
\hline 13 & 175742.87 & -4689.1 & 15.33 & -0.53 & 70 & 180 & - & - \\
\hline 14 & 173643.13 & -432438.3 & 15.17 & -0.52 & 340 & 600 & - & - \\
\hline 15 & 175318.12 & -43139.5 & 15.80 & -0.50 & 110 & 700 & - & late \\
\hline 16 & 175557.78 & -452437.9 & 10.04 & -0.50 & & & & \\
\hline 17 & 174652.66 & -453255.7 & 14.93 & -0.50 & 1300 & 2400 & composite & late? \\
\hline 18 & 175432.12 & -454152.0 & 12.22 & -0.49 & 4500 & 8500 & mid-B & mid-B \\
\hline 19 & 17392.20 & -45038.7 & 13.10 & -0.48 & 670 & 2800 & early-B & - \\
\hline 20 & 174352.38 & -44373.3 & 15.55 & -0.48 & 1500 & 1400 & mid-B & $\mathrm{AB} ?$ \\
\hline 21 & 17474.87 & -43139.0 & 10.24 & -0.45 & & & & \\
\hline 22 & 174754.89 & -425233.3 & 14.06 & -0.44 & 1800 & 3600 & mid-B & mid-B \\
\hline 23 & 173326.01 & -45937.0 & 13.48 & -0.44 & 1400 & 2300 & early-B & $\mathrm{AB} ?$ \\
\hline 24 & 175530.85 & -461321.4 & 10.01 & -0.43 & & & & \\
\hline 25 & 18054.42 & -453113.6 & 12.73 & -0.42 & 1500 & 2200 & $\mathrm{AB}$ & $\mathrm{AB} ?$ \\
\hline 26 & 175116.41 & -444729.1 & 12.86 & -0.42 & 1300 & 4700 & peculiar & - \\
\hline 27 & 174120.56 & -465447.3 & 11.10 & -0.41 & & & & \\
\hline 28 & 17501.30 & -472229.5 & 12.97 & -0.41 & 1500 & 900 & early-B & - \\
\hline 29 & 175546.15 & -462319.8 & 14.06 & -0.41 & 0 & 0 & - & - \\
\hline 30 & 17402.46 & $\begin{array}{lll}-43 & 13 & 1.1\end{array}$ & 10.78 & -0.41 & & & & \\
\hline 31 & 175719.03 & -465210.0 & 15.97 & -0.41 & 105 & 500 & - & - \\
\hline 32 & 175512.07 & -443548.6 & 10.01 & -0.40 & & & & \\
\hline 33 & 17414.97 & -47327.2 & 12.51 & -0.40 & & & & \\
\hline 34 & 17443.40 & -452155.2 & 13.93 & -0.39 & 2000 & 5200 & mid-B & $\mathrm{AB} ?$ \\
\hline 35 & 175851.41 & -45211.5 & 15.10 & -0.39 & 600 & 600 & $\mathrm{AB} ?$ & - \\
\hline 36 & 173811.76 & -445639.6 & 10.84 & -0.38 & & & & \\
\hline 37 & 174317.12 & -451034.1 & 10.32 & -0.38 & & & & \\
\hline 38 & 175156.51 & -4383.5 & 11.20 & -0.37 & 24000 & 39000 & mid-B & $\mathrm{AB}$ \\
\hline 39 & 17544.23 & -444944.5 & 13.06 & -0.36 & 4400 & 6600 & late-B & $\mathrm{AB}$ \\
\hline 40 & 173649.31 & -434128.9 & 10.31 & -0.36 & & & & \\
\hline 41 & 174429.94 & -443848.6 & 10.17 & -0.35 & & & & \\
\hline 42 & 174837.49 & -442519.7 & 14.56 & -0.35 & 1300 & 3200 & mid-B & $\mathrm{AB}$ \\
\hline 43 & 175457.58 & -43148.3 & 10.65 & -0.35 & & & & \\
\hline 44 & 175655.95 & -454151.1 & 10.46 & -0.34 & & & & \\
\hline 45 & 173556.22 & -45116.9 & 10.35 & -0.34 & & & & \\
\hline 46 & 175054.19 & -4581.2 & 12.05 & -0.34 & 7000 & 15000 & late-B & $\mathrm{AB}$ \\
\hline 47 & 174851.02 & $\begin{array}{lll}-43 & 18 & 4.4\end{array}$ & 13.81 & -0.34 & 0 & 0 & - & - \\
\hline 48 & 173718.41 & -464310.3 & 10.40 & -0.34 & & & & \\
\hline 49 & 173620.18 & -46383.3 & 11.53 & -0.34 & 8000 & 300 & mid-B & - \\
\hline 50 & 175652.15 & $\begin{array}{lll}-45 & 29 & 0.1\end{array}$ & 12.52 & -0.33 & 400 & 140 & - & - \\
\hline 51 & 174724.99 & -463027.0 & 13.53 & -0.33 & 3100 & 2800 & mid-B & $\mathrm{AB}$ \\
\hline 52 & 173736.18 & -4628.2 & 13.16 & -0.32 & 860 & 0 & - & - \\
\hline 53 & 174320.65 & -471651.2 & 10.53 & -0.32 & & & & \\
\hline 54 & 173751.87 & -461925.1 & 10.43 & -0.32 & & & & \\
\hline 55 & 17353.68 & -45398.2 & 11.04 & -0.32 & 18000 & 1800 & late-B & - \\
\hline 56 & 174311.81 & -433420.9 & 10.90 & -0.32 & & & & \\
\hline 57 & 174149.77 & -441430.6 & 11.61 & -0.32 & 4200 & 6300 & late-B & $\mathrm{AB}$ \\
\hline 58 & 174321.52 & -431955.5 & 11.81 & -0.31 & 32000 & 51000 & late-B & $\mathrm{AB}$ \\
\hline
\end{tabular}


Table 3. Blue stellar candidates for Field 333.

\begin{tabular}{|c|c|c|c|c|c|c|c|c|}
\hline Star & RA (2000) & Dec & $V$ & $(u-v)$ & $C_{\mathrm{b}}$ & $\overline{C_{\mathrm{r}}}$ & Blue spectra & Red spectra \\
\hline 1 & 172242.22 & -391026.5 & 15.44 & -1.48 & 2400 & 800 & late-B? & - \\
\hline 2 & 17328.87 & -40137.3 & 14.07 & -1.08 & 1700 & 0 & early-B & - \\
\hline 3 & 172231.16 & -3889.2 & 15.60 & -0.98 & 700 & 1100 & peculiar & $\mathrm{PN}$ \\
\hline 4 & 173453.86 & -403816.8 & 14.81 & -0.96 & 1700 & 900 & mid-B & $\mathrm{AB}$ \\
\hline 5 & 17239.15 & -404716.8 & 15.02 & -0.94 & 610 & 1200 & $\mathrm{AB} ?$ & late? \\
\hline 6 & 172555.39 & -40587.2 & 13.78 & -0.86 & 4100 & 5100 & $\operatorname{sdB}$ & peculiar \\
\hline 7 & 172910.95 & -403430.9 & 15.85 & -0.81 & 710 & 900 & late? & late? \\
\hline 8 & 17344.39 & -392341.4 & 10.99 & -0.61 & 21000 & 26000 & peculiar & PN \\
\hline 9 & $17 \quad 15 \quad 23.44$ & -38120.8 & 14.80 & -0.52 & & & & \\
\hline 10 & 17273.27 & -384755.4 & 13.75 & -0.50 & 6200 & 6800 & late & late? \\
\hline 11 & 17137.32 & -384734.5 & 11.85 & -0.44 & 4300 & 6700 & early-B & $\mathrm{AB} ?$ \\
\hline 12 & 171333.44 & $\begin{array}{llll}-39 & 0 & 38.4\end{array}$ & 10.15 & -0.41 & 8600 & 7400 & late-B & $\mathrm{AB}$ \\
\hline 13 & 172921.50 & $-3831 \quad 12.3$ & 13.74 & -0.41 & & & & \\
\hline 14 & 172852.49 & -37573.7 & 11.98 & -0.40 & 15000 & 17000 & late-B & $\mathrm{AB}$ \\
\hline 15 & 17263.76 & -382320.7 & 11.74 & -0.38 & 18000 & 12000 & late-B & $\mathrm{AB}$ \\
\hline 16 & 173347.11 & -384728.4 & 14.11 & -0.38 & 800 & 75 & late & - \\
\hline 17 & 172327.20 & -374430.7 & 12.21 & -0.37 & 12000 & 13000 & mid-B & mid-B \\
\hline 18 & 172222.41 & -383331.3 & 12.65 & -0.37 & 4900 & 6700 & late-B & $\mathrm{AB} ?$ \\
\hline 19 & 173112.47 & -38433.9 & 11.21 & -0.37 & 25000 & 9200 & mid-B & $\mathrm{AB}$ \\
\hline 20 & 172656.38 & $-38 \quad 1427.1$ & 10.43 & -0.37 & & & & \\
\hline 21 & 172312.94 & -375927.4 & 12.44 & -0.36 & 9700 & 7400 & late & $\mathrm{AB} ?$ \\
\hline 22 & 172544.27 & -382916.8 & 12.41 & -0.36 & 18000 & 16000 & late-B & $\mathrm{AB}$ \\
\hline 23 & 172242.60 & -38421.5 & 13.17 & -0.36 & 5400 & 8500 & mid-B & $\mathrm{AB}$ \\
\hline 24 & 171314.80 & -382537.3 & 11.81 & -0.36 & 29000 & 13000 & late-B & $\mathrm{AB}$ \\
\hline 25 & 172857.82 & -374445.4 & 10.76 & -0.35 & & & & \\
\hline 26 & 17160.35 & -381234.3 & 11.62 & -0.35 & 30000 & 13000 & late-B & $\mathrm{AB}$ \\
\hline 27 & 171236.66 & -38472.3 & 11.48 & -0.35 & 30000 & 15000 & $\mathrm{AB}$ & $\mathrm{AB}$ \\
\hline 28 & 172154.11 & -383122.0 & 10.96 & -0.35 & & & & \\
\hline 29 & 17272.01 & -38557.5 & 10.46 & -0.35 & & & & \\
\hline 30 & 173423.97 & -383536.5 & 13.45 & -0.34 & 22000 & 10000 & late & late? \\
\hline 31 & 172648.53 & -401433.8 & 10.70 & -0.34 & & & & \\
\hline 32 & 171628.28 & -38492.2 & 10.25 & -0.34 & & & & \\
\hline 33 & 172822.70 & -384651.7 & 11.46 & -0.34 & 39000 & 28000 & mid-B & $\mathrm{AB}$ \\
\hline 34 & 172234.03 & -38256.6 & 13.22 & -0.34 & 4400 & 6300 & mid-B & $\mathrm{AB}$ \\
\hline 35 & 173440.59 & -41248.4 & 15.44 & -0.33 & 900 & 1000 & late & late? \\
\hline 36 & 172324.33 & -375748.4 & 10.55 & -0.33 & & & & \\
\hline 37 & 173341.66 & -385428.1 & 13.19 & -0.33 & 2200 & 1100 & late & late? \\
\hline 38 & 173353.48 & -405324.2 & 10.49 & -0.33 & 2700 & 0 & $\mathrm{AB}$ & - \\
\hline 39 & 172337.00 & -405826.1 & 10.39 & -0.33 & & & & \\
\hline 40 & 17347.46 & -404634.5 & 10.34 & -0.33 & & & & \\
\hline 41 & 172619.31 & -38845.5 & 11.19 & -0.33 & & & & \\
\hline 42 & 172551.63 & -381959.8 & 12.33 & -0.32 & & & & \\
\hline 43 & 172131.77 & -385122.8 & 11.26 & -0.32 & 22000 & 12000 & early-B & early-B \\
\hline 44 & 172557.11 & -38647.0 & 12.22 & -0.32 & 13000 & 12000 & late-B & $\mathrm{AB}$ \\
\hline 45 & 173415.45 & -395534.6 & 10.62 & -0.32 & & & & \\
\hline 46 & 173049.31 & -38428.9 & 13.86 & -0.32 & 1100 & 420 & late & - \\
\hline 47 & 172227.19 & -382858.7 & 12.98 & -0.32 & & & & \\
\hline 48 & 172049.47 & -38509.8 & 11.56 & -0.32 & 23000 & 21000 & mid-B & $\mathrm{AB}$ \\
\hline 49 & 172310.22 & -382556.3 & 11.94 & -0.32 & 13000 & 20000 & early-B & early-B \\
\hline 50 & 172345.69 & -395357.1 & 10.28 & -0.32 & & & & \\
\hline 51 & 172345.27 & -3852.4 & 13.12 & -0.32 & 4500 & 8300 & $\mathrm{AB}$ & $\mathrm{AB}$ \\
\hline 52 & 173353.48 & -38383.3 & 12.91 & -0.32 & 520 & 40 & $\mathrm{AB}$ & - \\
\hline 53 & 172714.80 & -40534.2 & 10.44 & -0.32 & & & & \\
\hline 54 & 172819.35 & -38427.5 & 15.07 & -0.32 & & & & \\
\hline 55 & 17224.98 & -382657.9 & 12.87 & -0.32 & 5500 & 3900 & late-B & $\mathrm{AB}$ \\
\hline 56 & 173617.98 & -403659.0 & 10.40 & -0.32 & 58000 & 72000 & mid-B & $\mathrm{AB}$ \\
\hline 57 & 173259.99 & -391620.3 & 11.19 & -0.31 & 11000 & 2400 & mid-B & $\mathrm{AB}$ \\
\hline 58 & 173115.47 & -375751.1 & 12.27 & -0.31 & & & & \\
\hline 59 & 171518.77 & -38848.0 & 10.31 & -0.31 & & & & \\
\hline 60 & 173153.14 & -38955.3 & 11.60 & -0.31 & 15000 & 10000 & early-B & early-B \\
\hline 61 & 17279.68 & -405511.5 & 12.85 & -0.31 & 6300 & 9000 & mid-B & $\mathrm{AB}$ \\
\hline
\end{tabular}


Table 4. Blue stellar candidates for Field 391.

\begin{tabular}{|c|c|c|c|c|c|c|c|c|}
\hline Star & RA (2000) & Dec & $\bar{V}$ & $(u-v)$ & $C_{\mathrm{b}}$ & $C_{\mathrm{r}}$ & Blue spectra & Red spectra \\
\hline 1 & 165935.64 & -33930.3 & 15.09 & -0.93 & & & & \\
\hline 2 & 164322.62 & -33425.9 & 13.13 & -0.86 & 0 & 0 & - & - \\
\hline 3 & 165541.93 & -353718.4 & 15.47 & -0.82 & 0 & 0 & - & - \\
\hline 4 & 16575.36 & -33140.5 & 15.49 & -0.74 & 2200 & 2800 & early-B & late? \\
\hline 5 & 163951.80 & -344232.9 & 15.41 & -0.73 & 0 & 0 & - & - \\
\hline 6 & 165340.12 & -332041.7 & 13.93 & -0.64 & 4200 & 12000 & late & late \\
\hline 7 & 16555.82 & -34304.8 & 14.35 & -0.60 & 4700 & 1200 & late & - \\
\hline 8 & 164735.98 & $\begin{array}{lll}-33 & 10 & 11.4\end{array}$ & 14.53 & -0.54 & 800 & 450 & early-B & - \\
\hline 9 & 165450.83 & -341610.3 & 13.49 & -0.54 & 13000 & 15000 & early-B & early-B \\
\hline 10 & 165749.15 & -332119.1 & 14.60 & -0.50 & 4100 & 4800 & late-B & $\mathrm{AB}$ \\
\hline 11 & 165112.16 & -334721.3 & 13.75 & -0.47 & 9500 & 4800 & early-B & early-B \\
\hline 12 & 17228.10 & -34216.6 & 11.36 & -0.45 & 22000 & 4400 & late-B & $\mathrm{AB}$ \\
\hline 13 & 165645.34 & -335949.7 & 15.27 & -0.44 & 3500 & 2600 & early-B & early-B \\
\hline 14 & 165329.02 & -37325.5 & 10.42 & -0.43 & & & & \\
\hline 15 & 17055.07 & -3486.6 & 11.24 & -0.43 & 54000 & 14000 & mid-B & $\mathrm{AB}$ \\
\hline 16 & 17230.66 & -351531.9 & 11.02 & -0.42 & 18000 & 2700 & early-B & $\mathrm{AB}$ ? \\
\hline 17 & 165620.49 & -37928.4 & 10.10 & -0.41 & & & & \\
\hline 18 & 165546.31 & -342022.1 & 14.74 & -0.40 & 5900 & 3900 & mid-B & $\mathrm{AB}$ \\
\hline 19 & 165711.52 & -361039.5 & 10.52 & -0.40 & & & & \\
\hline 20 & 165015.97 & -37636.4 & 10.53 & -0.39 & & & & \\
\hline 21 & 17145.37 & -34718.3 & 11.98 & -0.39 & 17000 & 11000 & late-B & $\mathrm{AB}$ \\
\hline 22 & 17246.60 & -344029.0 & 15.94 & -0.39 & & & & \\
\hline 23 & 165918.33 & -332535.2 & 11.98 & -0.39 & 42000 & 3900 & early-B & early-B \\
\hline 24 & 165536.27 & -331936.1 & 14.72 & -0.38 & & & & \\
\hline 25 & 165852.04 & -341319.7 & 10.91 & -0.38 & & & & \\
\hline 26 & 165828.38 & -332114.6 & 11.65 & -0.38 & 54000 & 53000 & late-B & $\mathrm{AB}$ \\
\hline 27 & 17134.49 & -352233.1 & 10.84 & -0.38 & & & & \\
\hline 28 & 17159.45 & -343112.7 & 10.68 & -0.37 & & & & \\
\hline 29 & 165938.86 & -363248.5 & 11.87 & -0.36 & 1800 & 360 & $\mathrm{AB}$ & - \\
\hline 30 & 16588.25 & -36399.8 & 10.31 & -0.35 & & & & \\
\hline 31 & 165238.80 & $-3647 \quad 18.5$ & 11.28 & -0.34 & 1000 & 450 & $\mathrm{AB}$ & - \\
\hline 32 & 165640.77 & -334349.9 & 12.27 & -0.34 & & & & \\
\hline 33 & 165457.30 & -332150.1 & 10.98 & -0.34 & & & & \\
\hline 34 & 165429.45 & -33142.2 & 13.45 & -0.33 & 8000 & 3300 & late-B & $\mathrm{AB} ?$ \\
\hline 35 & 164020.24 & -35521.4 & 14.89 & -0.32 & & & & \\
\hline 36 & 165454.31 & -333030.1 & 11.29 & -0.32 & & & & \\
\hline 37 & 165732.39 & -332710.8 & 11.91 & -0.32 & & & & \\
\hline 38 & 17043.41 & -334814.7 & 11.54 & -0.32 & 18000 & 21000 & late-B & $\mathrm{AB}$ \\
\hline 39 & 17352.96 & -35222.3 & 12.61 & -0.32 & 5800 & 3000 & late-B & $\mathrm{AB}$ ? \\
\hline 40 & 165838.76 & -341011.3 & 15.54 & -0.31 & 1800 & 1100 & late & late? \\
\hline 41 & 165726.87 & -325121.8 & 13.40 & -0.31 & 4600 & 120 & late & - \\
\hline 42 & 17121.22 & -353812.9 & 12.22 & -0.30 & 1000 & 240 & $\mathrm{AB}$ & - \\
\hline 43 & 165432.61 & -372254.2 & 13.25 & -0.30 & 590 & 330 & late? & - \\
\hline 44 & 17350.31 & -345058.5 & 11.43 & -0.29 & 18000 & 11000 & late-B & $\mathrm{AB}$ \\
\hline 45 & 17320.33 & -355041.6 & 12.19 & -0.29 & 2000 & 510 & $\mathrm{AB} ?$ & PN? \\
\hline 46 & 165635.14 & -363728.5 & 11.80 & -0.29 & 720 & 180 & $\mathrm{AB}$ & - \\
\hline 47 & 164156.15 & -343140.8 & 12.48 & -0.29 & 880 & 170 & $\mathrm{AB} ?$ & - \\
\hline 48 & 165845.62 & -362323.9 & 11.99 & -0.29 & 2600 & 330 & $\mathrm{AB}$ & - \\
\hline 49 & 165427.83 & -33524.3 & 11.10 & -0.29 & 130000 & 66000 & late-B & $\mathrm{AB}$ \\
\hline 50 & 165730.49 & -333357.8 & 10.97 & -0.29 & & & & \\
\hline 51 & 165734.72 & -325458.8 & 12.09 & -0.29 & & & & \\
\hline 52 & 165457.23 & -33243.0 & 13.88 & -0.29 & & & & \\
\hline 53 & 165114.46 & -355347.1 & 10.57 & -0.29 & & & & \\
\hline 54 & 17038.72 & -344855.2 & 11.84 & -0.29 & 5900 & 1400 & $\mathrm{AB}$ & $\mathrm{AB} ?$ \\
\hline 55 & 165947.98 & -34439.7 & 11.59 & -0.28 & 35000 & 10000 & $\mathrm{sdB}$ & $\mathrm{AB}$ \\
\hline 56 & 164651.23 & -334323.3 & 15.42 & -0.28 & & & & \\
\hline 57 & 17341.30 & $\begin{array}{lll}-35 & 30 & 0.8\end{array}$ & 11.27 & -0.28 & 5600 & 990 & early-B & $\mathrm{AB} ?$ \\
\hline 58 & 165737.07 & -33145.9 & 13.89 & -0.28 & & & & \\
\hline 59 & 165521.07 & -323910.1 & 15.44 & -0.28 & & & & \\
\hline 60 & 17021.82 & -344149.9 & 11.11 & -0.28 & & & & \\
\hline 61 & 17150.53 & -341228.1 & 10.51 & -0.27 & & & & \\
\hline 62 & 17045.72 & -362221.1 & 11.74 & -0.27 & 1300 & 240 & $\mathrm{AB}$ & - \\
\hline 63 & 16579.93 & -36343.9 & 11.10 & -0.27 & 20000 & 1600 & mid-B & - \\
\hline 64 & 17323.38 & -35228.4 & 12.48 & -0.27 & 5400 & 2000 & late-B & $\mathrm{AB} ?$ \\
\hline
\end{tabular}


Table 5. Blue stellar candidates for Field 520.

\begin{tabular}{|c|c|c|c|c|c|c|c|c|}
\hline Star & RA (2000) & Dec & $V$ & $(u-v)$ & $C_{\mathrm{b}}$ & $C_{\mathrm{r}}$ & Blue spectra & Red spectra \\
\hline 1 & 173833.49 & $-24 \quad 1530.8$ & 15.41 & -1.43 & 2800 & 5200 & early-B & early-B \\
\hline 2 & 174547.56 & -261026.9 & 14.37 & -1.01 & & & & \\
\hline 3 & 174454.37 & $-26 \quad 6 \quad 0.5$ & 15.22 & -0.99 & 280 & 280 & - & PN? \\
\hline 4 & 174015.68 & -232846.4 & 12.33 & -0.63 & 1200 & 4000 & $\mathrm{AB}$ & early? -B \\
\hline 5 & 174151.08 & -224522.7 & 14.68 & -0.60 & 1300 & 2600 & early-B & early-B \\
\hline 6 & 174850.88 & -251020.2 & 12.33 & -0.53 & 1200 & 2200 & $\mathrm{AB}$ & $\mathrm{AB}$ \\
\hline 7 & 174150.50 & -225957.2 & 12.74 & -0.50 & 500 & 2600 & $\mathrm{AB} ?$ & $\mathrm{AB}$ \\
\hline 8 & 174038.35 & -231259.4 & 14.13 & -0.49 & 350 & 1100 & - & - \\
\hline 9 & 174552.46 & -26849.0 & 12.81 & -0.47 & 140 & 710 & PN? & $\mathrm{PN}$ \\
\hline 10 & 173824.12 & -224838.7 & 14.56 & -0.46 & 1200 & 2200 & early-B & early-B? \\
\hline 11 & 17452.31 & -262756.8 & 11.54 & -0.46 & 600 & 1000 & $\mathrm{AB}$ & - \\
\hline 12 & 174959.54 & -251729.0 & 15.52 & -0.45 & 70 & 20 & - & - \\
\hline 13 & 173326.43 & -232049.6 & 15.48 & -0.42 & 680 & 4000 & late & late \\
\hline 14 & 174341.83 & -24946.9 & 15.43 & -0.42 & 350 & 4000 & - & late \\
\hline 15 & 173949.34 & -23226.7 & 14.84 & -0.42 & 910 & 5200 & early-B & early-B? \\
\hline 16 & 174813.31 & -253855.1 & 14.69 & -0.42 & & & & \\
\hline 17 & 17457.88 & -261912.3 & 12.50 & -0.42 & & & & \\
\hline 18 & 174858.80 & -254523.8 & 14.65 & -0.41 & & & & \\
\hline 19 & 174536.13 & -225741.8 & 12.24 & -0.41 & 4800 & 13000 & $\mathrm{AB}$ & late? \\
\hline 20 & 174951.84 & -254219.0 & 13.18 & -0.41 & 210 & 360 & - & - \\
\hline 21 & 17494.26 & -252630.6 & 11.73 & -0.40 & & & & \\
\hline 22 & 174935.48 & -26220.0 & 11.43 & -0.40 & 1000 & 1380 & mid-B? & $\mathrm{AB}$ \\
\hline 23 & 17501.26 & -253810.8 & 15.63 & -0.40 & & & & \\
\hline 24 & 174842.81 & -254024.3 & 13.63 & -0.40 & 90 & 1000 & $\mathrm{~B} ?$ & late? \\
\hline 25 & 174756.38 & -262039.7 & 14.75 & -0.39 & & & & \\
\hline 26 & 174757.76 & -261930.4 & 14.57 & -0.39 & 70 & 600 & - & late \\
\hline 27 & 17472.62 & -262810.8 & 15.41 & -0.39 & 70 & 80 & - & - \\
\hline 28 & 174943.91 & -255028.6 & 15.93 & -0.39 & & & & \\
\hline 29 & 17493.98 & -251456.7 & 15.25 & -0.39 & & & & \\
\hline 30 & 174736.54 & -25292.9 & 11.31 & -0.39 & 1200 & 1400 & early-B? & $\mathrm{AB} ?$ \\
\hline 31 & 174740.97 & -255721.7 & 15.51 & -0.38 & & & & \\
\hline 32 & 174814.18 & -262432.6 & 15.15 & -0.38 & & & & \\
\hline 33 & 174942.61 & -253241.8 & 12.92 & -0.38 & 200 & 100 & - & - \\
\hline 34 & 17462.39 & -27246.4 & 15.12 & -0.38 & & & & \\
\hline 35 & 174256.78 & -263334.4 & 12.18 & -0.38 & 80 & 320 & - & - \\
\hline 36 & 174318.02 & -225948.7 & 15.60 & -0.38 & 350 & 1400 & - & late? \\
\hline 37 & 174930.84 & -253536.1 & 15.00 & -0.38 & & & & \\
\hline 38 & 174917.93 & -251628.6 & 15.46 & -0.38 & & & & \\
\hline 39 & 174744.10 & -262046.2 & 12.50 & -0.38 & 900 & 1200 & $\mathrm{AB}$ & $\mathrm{AB}$ \\
\hline 40 & 174818.45 & -26209.7 & 14.86 & -0.37 & & & & \\
\hline 41 & 174846.23 & -255310.4 & 13.63 & -0.37 & & & & \\
\hline 42 & 174747.07 & -26376.4 & 14.27 & -0.37 & 150 & 40 & - & - \\
\hline 43 & 174559.77 & -2672.2 & 11.57 & -0.37 & & & & \\
\hline 44 & 173856.79 & -22476.1 & 15.75 & -0.37 & & & & \\
\hline 45 & 174813.68 & -262033.9 & 11.81 & -0.37 & & & & \\
\hline 46 & 174542.73 & -225626.2 & 12.65 & -0.37 & & & & \\
\hline 47 & 174956.27 & -253324.2 & 12.97 & -0.36 & & & & \\
\hline 48 & 17508.28 & -254458.2 & 15.52 & -0.36 & & & & \\
\hline 49 & 174610.84 & -27355.3 & 15.15 & -0.36 & 60 & 100 & - & - \\
\hline 50 & 173517.71 & -231858.5 & 13.73 & -0.35 & 1800 & 1100 & late & late \\
\hline 51 & 17483.24 & -2607.1 & 15.83 & -0.35 & & & & \\
\hline 52 & 174617.89 & -26127.9 & 12.50 & -0.35 & 200 & 360 & - & - \\
\hline 53 & 175012.40 & -252429.3 & 11.85 & -0.35 & 1500 & 2100 & early-B & early-B \\
\hline 54 & 174910.32 & -253613.3 & 12.53 & -0.34 & & & & \\
\hline 55 & 17453.55 & -242223.9 & 11.72 & -0.34 & 300 & 920 & - & - \\
\hline 56 & 173426.16 & -2310.2 & 13.69 & -0.34 & 6400 & 4900 & late & $\mathrm{AB}$ \\
\hline 57 & 174754.33 & -244631.5 & 11.95 & -0.34 & 140 & 250 & - & - \\
\hline 58 & 174745.26 & -262044.7 & 15.63 & -0.34 & & & & \\
\hline 59 & 174817.88 & -26211.6 & 11.43 & -0.34 & & & & \\
\hline 60 & 174530.83 & -265422.3 & 11.85 & -0.34 & 290 & 240 & $\mathrm{AB}$ & - \\
\hline 61 & 174143.67 & -231830.3 & 14.43 & -0.34 & 1000 & 400 & late? & late? \\
\hline 62 & 174917.02 & -26931.5 & 15.28 & -0.34 & & & & \\
\hline 63 & 174547.55 & $\begin{array}{lll}-26 & 11 & 0.0\end{array}$ & 14.21 & -0.34 & & & & \\
\hline 64 & 174835.14 & -253226.7 & 12.43 & -0.33 & 140 & 40 & - & - \\
\hline
\end{tabular}


Table 6. Blue stellar candidates for Field 522.

\begin{tabular}{|c|c|c|c|c|c|c|c|c|}
\hline Star & RA (2000) & Dec & $V$ & $(u-v)$ & $C_{\mathrm{b}}$ & $C_{\mathrm{r}}$ & Blue spectra & Red spectra \\
\hline 1 & 18207.90 & -244710.3 & 15.87 & -0.84 & 910 & 4400 & late & late \\
\hline 2 & 182032.42 & -264711.2 & 15.92 & -0.77 & 0 & 140 & - & - \\
\hline 3 & 18208.87 & -24155.3 & 14.98 & -0.60 & 1000 & 1900 & $\mathrm{PN}$ & $\mathrm{PN}$ \\
\hline 4 & 18244.07 & -26745.0 & 10.11 & -0.55 & & & & \\
\hline 5 & 183129.34 & -241448.0 & 12.91 & -0.54 & 0 & 0 & - & - \\
\hline 6 & 183231.47 & -234351.5 & 13.43 & -0.52 & 0 & 0 & - & - \\
\hline 7 & 182820.20 & -233512.6 & 12.62 & -0.45 & 150 & 0 & - & - \\
\hline 8 & 18268.35 & -271135.3 & 15.77 & -0.45 & 1100 & 2000 & $\mathrm{AB}$ & $\mathrm{AB}$ \\
\hline 9 & 181822.44 & -25657.9 & 10.58 & -0.45 & & & & \\
\hline 10 & 182439.94 & -263229.7 & 13.28 & -0.44 & 18000 & 24000 & mid-B & $\mathrm{AB}$ \\
\hline 11 & 182450.60 & -261048.1 & 11.86 & -0.41 & 76000 & 81000 & early-B & early-B \\
\hline 12 & 182146.40 & $-23 \quad 1026.1$ & 10.49 & -0.41 & & & & \\
\hline 13 & 181829.13 & -255444.9 & 13.64 & -0.40 & 1300 & 1500 & $\mathrm{AB}$ & late? \\
\hline 14 & 182532.40 & -235343.5 & 12.99 & -0.39 & 500 & 600 & late & - \\
\hline 15 & 183348.83 & -243136.4 & 12.45 & -0.38 & 1200 & 1500 & early-B & late? \\
\hline 16 & 181919.77 & -235220.0 & 11.03 & -0.38 & 1300 & 400 & early-B & - \\
\hline 17 & 182326.78 & -232832.7 & 12.61 & -0.38 & 630 & 250 & early-B? & - \\
\hline 18 & 18288.68 & -27040.8 & 15.23 & -0.37 & 1100 & 2200 & late-B & $\mathrm{AB}$ \\
\hline 19 & 182220.46 & -263428.6 & 10.13 & -0.37 & & & & \\
\hline 20 & 182158.06 & -242035.7 & 10.89 & -0.37 & & & & \\
\hline 21 & 181816.93 & -25822.0 & 13.79 & -0.36 & 1900 & 770 & mid-B & - \\
\hline 22 & 182729.48 & -265743.9 & 10.40 & -0.35 & & & & \\
\hline 23 & 182457.44 & -252029.8 & 14.15 & -0.35 & 12000 & 12000 & early-B & early-B \\
\hline 24 & 181844.25 & -225850.1 & 10.06 & -0.35 & & & & \\
\hline 25 & 182449.71 & -22479.5 & 13.77 & -0.35 & & & & \\
\hline 26 & 182225.85 & -223711.9 & 15.12 & -0.33 & 0 & 0 & - & - \\
\hline 27 & 181830.97 & -233351.4 & 11.02 & -0.33 & 910 & 830 & mid-B & - \\
\hline 28 & 181832.22 & -23832.0 & 10.28 & -0.33 & & & & \\
\hline 29 & 18249.86 & -23226.8 & 10.49 & -0.33 & & & & \\
\hline 30 & 182656.28 & -263420.0 & 15.82 & -0.33 & 1100 & 2600 & $\mathrm{AB}$ & $\mathrm{AB}$ \\
\hline 31 & 181726.01 & -245824.0 & 14.58 & -0.32 & 510 & 1700 & late & late? \\
\hline 32 & 182126.71 & -224757.0 & 10.24 & -0.32 & 60 & 70 & - & PN? \\
\hline 33 & 182441.51 & -22406.3 & 15.50 & -0.32 & & & & \\
\hline 34 & 181453.80 & -264554.0 & 12.03 & -0.32 & 800 & 970 & $\mathrm{AB}$ & $\mathrm{AB}$ \\
\hline 35 & 182853.92 & -264530.7 & 14.32 & -0.32 & 730 & 4200 & late & late? \\
\hline 36 & 18184.42 & -2533.9 & 14.02 & -0.31 & 1200 & 880 & $\mathrm{AB}$ & - \\
\hline 37 & 182452.49 & $-22 \quad 4612.4$ & 12.51 & -0.31 & 290 & 460 & - & - \\
\hline 38 & 181652.10 & -231355.4 & 11.83 & -0.31 & & & & \\
\hline 39 & 182315.50 & -272256.4 & 14.53 & -0.31 & 450 & 880 & $\mathrm{AB}$ & late? \\
\hline 40 & $1817 \quad 1.12$ & -22502.4 & 11.04 & -0.31 & 190 & 300 & - & - \\
\hline 41 & 183138.04 & -244353.6 & 10.22 & -0.30 & & & & \\
\hline 42 & 182631.13 & -26841.3 & 13.65 & -0.30 & 4200 & 6600 & late-B & $\mathrm{AB}$ \\
\hline 43 & 182834.44 & -235834.4 & 11.18 & -0.30 & 1800 & 380 & early-B & - \\
\hline 44 & 182836.87 & -22464.3 & 15.27 & -0.30 & & & & \\
\hline 45 & 181932.75 & -231529.6 & 11.83 & -0.30 & 230 & 510 & - & PN? \\
\hline 46 & 181637.98 & -242737.1 & 11.18 & -0.30 & 2000 & 960 & $\mathrm{AB}$ & - \\
\hline 47 & 183050.42 & -24215.5 & 11.08 & -0.30 & 690 & 660 & late-B? & - \\
\hline 48 & 182948.85 & -231152.3 & 10.82 & -0.29 & & & & \\
\hline 49 & 181453.34 & -23 196.9 & 10.99 & -0.29 & & & & \\
\hline 50 & 182017.39 & -254326.9 & 14.69 & -0.29 & 1600 & 2500 & mid-B & $\mathrm{AB} ?$ \\
\hline 51 & 181629.11 & -23830.1 & 11.68 & -0.29 & 880 & 760 & $\mathrm{AB}$ & - \\
\hline 52 & 181740.84 & -253245.5 & 12.15 & -0.29 & 47000 & 31000 & mid-B & - \\
\hline 53 & 182018.60 & -23173.5 & 15.61 & -0.29 & & & & \\
\hline 54 & 183217.09 & -233644.7 & 12.62 & -0.29 & 0 & 0 & - & - \\
\hline 55 & 18322.97 & -234119.2 & 11.35 & -0.29 & & & & \\
\hline 56 & 181926.69 & -262132.1 & 11.51 & -0.29 & 4200 & 3700 & early-B & $\mathrm{AB}$ \\
\hline 57 & 182336.89 & -223622.1 & 12.18 & -0.29 & 110 & 70 & - & $\mathrm{PN}$ \\
\hline 58 & 182145.15 & -234627.8 & 11.72 & -0.28 & 2000 & 200 & $\mathrm{AB}$ & - \\
\hline 59 & 18201.93 & -225328.7 & 10.91 & -0.28 & & & & \\
\hline 60 & 181957.30 & -253549.3 & 15.78 & -0.28 & 4200 & 8100 & late & late? \\
\hline 61 & 181643.52 & -231727.3 & 14.38 & -0.28 & & & & \\
\hline 62 & 183144.36 & -251624.4 & 12.19 & -0.28 & 590 & 430 & $\mathrm{AB}$ & $\mathrm{AB} ?$ \\
\hline 63 & 182214.44 & -225138.7 & 12.21 & -0.28 & 180 & 0 & - & - \\
\hline
\end{tabular}


Table 7. Blue stellar candidates for Field 589.

\begin{tabular}{|c|c|c|c|c|c|c|c|c|}
\hline Star & RA (2000) & Dec & $V$ & $(u-v)$ & $C_{\mathrm{b}}$ & $\overline{C_{\mathrm{r}}}$ & Blue spectra & Red spectra \\
\hline 1 & 175151.12 & -20238.4 & 15.46 & -1.47 & 450 & 200 & sdB? & - \\
\hline 2 & $1746 \quad 17.13$ & -181234.6 & 15.85 & -1.39 & 70 & 0 & - & - \\
\hline 3 & 18014.23 & -18747.6 & 15.27 & -1.31 & 310 & 540 & - & late? \\
\hline 4 & $17 \quad 47 \quad 22.44$ & -193749.8 & 13.34 & -0.97 & 3100 & 200 & late-B & - \\
\hline 5 & 174736.47 & -19272.0 & 15.21 & -0.65 & 100 & 50 & - & - \\
\hline 6 & 1844.62 & -193830.1 & 11.71 & -0.52 & 12000 & 17000 & mid-B & early-B \\
\hline 7 & 174425.52 & -193754.1 & 12.02 & -0.51 & 2500 & 2400 & late & late? \\
\hline 8 & 175746.29 & -18329.8 & 12.15 & -0.49 & 0 & 0 & - & - \\
\hline 9 & 18448.03 & -193427.9 & 10.89 & -0.47 & & & & \\
\hline 10 & 18410.80 & -205732.4 & 12.08 & -0.47 & 5500 & 3100 & late-B & $\mathrm{AB}$ \\
\hline 11 & 18452.59 & -19574.0 & 12.67 & -0.45 & 70 & 0 & - & - \\
\hline 12 & 18243.51 & -204434.7 & 15.28 & -0.43 & & & & \\
\hline 13 & 18127.30 & -21401.7 & 12.20 & -0.42 & 7800 & 5900 & late-B & $\mathrm{AB}$ \\
\hline 14 & 18222.14 & -201136.6 & 11.93 & -0.41 & & & & \\
\hline 15 & 18415.79 & -201916.1 & 12.17 & -0.40 & 5100 & 1400 & late-B & $\mathrm{AB} ?$ \\
\hline 16 & 18432.70 & -204522.0 & 12.44 & -0.40 & 2200 & 700 & mid-B? & late? \\
\hline 17 & 1825.78 & -20913.2 & 11.70 & -0.40 & 1700 & 2000 & late & late \\
\hline 18 & 18430.38 & -20129.4 & 12.54 & -0.40 & & & & \\
\hline 19 & 1833.95 & -194524.4 & 11.71 & -0.40 & 13000 & 24000 & late-B & $\mathrm{AB}$ \\
\hline 20 & 18455.01 & -195541.6 & 11.55 & -0.39 & & & & \\
\hline 21 & 175958.20 & -19234.3 & 12.73 & -0.39 & 2100 & 540 & late-B & $\mathrm{AB}$ \\
\hline 22 & 175639.41 & -175642.9 & 14.81 & -0.39 & 300 & 200 & - & - \\
\hline 23 & 1828.55 & -193328.7 & 12.15 & -0.39 & 4500 & 11000 & late-B & $\mathrm{AB}$ \\
\hline 24 & 18435.88 & -195410.5 & 13.22 & -0.38 & & & & \\
\hline 25 & 18426.18 & -194223.3 & 12.12 & -0.38 & 3100 & 11000 & late-B & $\mathrm{AB}$ \\
\hline 26 & 18212.27 & -193741.5 & 12.31 & -0.38 & 1500 & 4500 & $\mathrm{AB}$ & late? \\
\hline 27 & 18452.33 & -194641.9 & 10.89 & -0.38 & & & & \\
\hline 28 & 17587.05 & -215221.6 & 15.97 & -0.38 & 460 & 0 & late & - \\
\hline 29 & 18235.54 & -204621.2 & 15.67 & -0.38 & & & & \\
\hline 30 & 175943.26 & -20222.7 & 12.30 & -0.38 & 10000 & 7800 & late & $\mathrm{AB}$ \\
\hline 31 & 18031.30 & -19 1942.8 & 11.53 & -0.37 & 7600 & 5100 & mid-B & $\mathrm{AB}$ \\
\hline 32 & 18310.47 & -20245.9 & 12.65 & -0.37 & 0 & 0 & - & - \\
\hline 33 & 174911.23 & -192950.3 & 12.26 & -0.37 & 2500 & 0 & early-B & - \\
\hline 34 & 175926.31 & -184733.4 & 14.70 & -0.36 & 0 & 0 & - & - \\
\hline 35 & 17570.34 & -201941.7 & 12.72 & -0.36 & 6800 & 3800 & late-B & $\mathrm{AB}$ \\
\hline 36 & 1807.95 & -182530.2 & 15.63 & -0.35 & & & & \\
\hline 37 & 18337.81 & -193015.8 & 11.92 & -0.35 & & & & \\
\hline 38 & 18230.96 & -21733.6 & 12.53 & -0.35 & 5600 & 10000 & $\mathrm{AB}$ & $\mathrm{AB}$ \\
\hline 39 & 174456.16 & -20241.0 & 13.70 & -0.35 & 1100 & 130 & $\mathrm{AB}$ & - \\
\hline 40 & 175840.07 & -175649.5 & 12.80 & -0.34 & 1700 & 410 & late-B & $\mathrm{AB} ?$ \\
\hline 41 & 175517.91 & -20502.3 & 12.67 & -0.34 & 3100 & 400 & $\mathrm{AB}$ & - \\
\hline 42 & 1847.01 & -204549.2 & 10.19 & -0.34 & & & & \\
\hline 43 & 18314.53 & -212220.6 & 11.88 & -0.34 & 2300 & 19000 & early-B & early-B \\
\hline 44 & 175912.03 & -22725.1 & 12.43 & -0.34 & 10000 & 1400 & late-B & $\mathrm{AB}$ \\
\hline 45 & 17558.81 & -202737.4 & 12.58 & -0.34 & 5500 & 290 & late-B & - \\
\hline 46 & 18017.21 & -184057.8 & 15.79 & -0.33 & & & & \\
\hline 47 & 1800.52 & -18 1630.1 & 15.82 & -0.33 & & & & \\
\hline 48 & 18350.39 & -19412.3 & 13.31 & -0.33 & & & & \\
\hline 49 & 18428.83 & -191854.3 & 13.55 & -0.33 & & & & \\
\hline 50 & 18010.93 & -183837.6 & 15.59 & -0.32 & & & & \\
\hline 51 & 175947.19 & -213822.8 & 12.67 & -0.32 & 10000 & 850 & late-B & - \\
\hline 52 & 175358.13 & -202625.3 & 12.34 & -0.32 & 0 & 0 & - & - \\
\hline 53 & 175921.19 & -20248.6 & 10.69 & -0.32 & 25000 & 2100 & early-B & early-B \\
\hline 54 & 17578.10 & -20186.0 & 12.17 & -0.32 & 10000 & 4900 & mid-B & $\mathrm{AB}$ \\
\hline 55 & 18154.79 & -19204.8 & 13.07 & -0.32 & & & & \\
\hline 56 & 175925.36 & -183512.7 & 15.26 & -0.32 & & & & \\
\hline 57 & 175536.52 & -175741.6 & 11.71 & -0.32 & 0 & 0 & - & - \\
\hline 58 & 18423.37 & -20716.1 & 13.48 & -0.32 & & & & \\
\hline 59 & 175842.09 & -19345.0 & 15.90 & -0.31 & & & & \\
\hline 60 & 18021.03 & -184525.2 & 12.93 & -0.31 & 1100 & 710 & late & late? \\
\hline 61 & 175338.83 & -201635.3 & 13.05 & -0.31 & 1600 & 300 & early-B & \\
\hline
\end{tabular}


Table 8. Blue stellar candidates for Field 591.

\begin{tabular}{|c|c|c|c|c|c|c|c|c|}
\hline Star & RA (2000) & Dec & $V$ & $(u-v)$ & $C_{\mathrm{b}}$ & $C_{\mathrm{r}}$ & Blue spectra & Red spectra \\
\hline 1 & 18342.47 & -22057.2 & 15.47 & -1.84 & & & & \\
\hline 2 & 183618.05 & -203859.2 & 15.28 & -0.98 & 1500 & 700 & $\mathrm{AB}$ & early-B? \\
\hline 3 & 184257.68 & -191219.2 & 15.41 & -0.81 & 250 & 240 & - & - \\
\hline 4 & 184316.61 & -183128.2 & 13.32 & -0.75 & 500 & 150 & late? & - \\
\hline 5 & 183935.58 & -191410.8 & 13.32 & -0.69 & 15000 & 17000 & early-B & early-B \\
\hline 6 & 184015.63 & -175824.7 & 15.39 & -0.65 & 490 & 270 & early-B? & $\mathrm{AB} ?$ \\
\hline 7 & 183215.56 & -213212.2 & 14.90 & -0.54 & 0 & 60 & - & - \\
\hline 8 & 184135.48 & -175248.9 & 12.41 & -0.53 & 17000 & 21000 & early-B & early-B \\
\hline 9 & 18385.25 & -212759.0 & 15.40 & -0.52 & 0 & 0 & - & - \\
\hline 10 & 18445.75 & -201149.1 & 15.94 & -0.51 & 80 & 110 & - & - \\
\hline 11 & 183311.50 & -214015.5 & 13.26 & -0.50 & & & & \\
\hline 12 & 184043.49 & -193330.7 & 11.93 & -0.50 & 23000 & 42000 & mid-B & mid-B \\
\hline 13 & 183536.86 & -22932.9 & 15.62 & -0.48 & 420 & 2200 & late & late? \\
\hline 14 & 18337.57 & -211616.2 & 13.08 & -0.48 & 1600 & 2500 & late & late? \\
\hline 15 & 184258.93 & -191131.3 & 13.32 & -0.47 & & & & \\
\hline 16 & 184431.99 & -205712.9 & 12.89 & -0.47 & 340 & 750 & late? & late? \\
\hline 17 & 183410.07 & -182640.1 & 15.77 & -0.47 & & & & \\
\hline 18 & 184025.49 & -18653.9 & 12.62 & -0.44 & 18000 & 23000 & mid-B & mid-B \\
\hline 19 & 184358.89 & -205242.6 & 12.80 & -0.42 & 280 & 140 & - & - \\
\hline 20 & 182918.63 & $\begin{array}{lll}-18 & 11 & 2.3\end{array}$ & 11.58 & -0.42 & 3400 & 2500 & early-B & $\mathrm{AB} ?$ \\
\hline 21 & 183734.80 & -205325.2 & 13.86 & -0.42 & 720 & 2600 & $\mathrm{PN}$ & $\mathrm{PN}$ \\
\hline 22 & 183844.28 & -214616.5 & 15.41 & -0.40 & 150 & 300 & $\mathrm{AB}$ & - \\
\hline 23 & 183435.67 & -205242.9 & 12.48 & -0.40 & 180 & 300 & $\mathrm{AB}$ & - \\
\hline 24 & 183647.89 & -20449.0 & 13.87 & -0.39 & 10000 & 13000 & mid-B & mid-B \\
\hline 25 & 184236.80 & -201417.3 & 11.12 & -0.39 & 36000 & 23000 & early-B & early-B \\
\hline 26 & 184033.32 & -201815.8 & 10.14 & -0.38 & & & & \\
\hline 27 & 184530.34 & -185030.3 & 10.02 & -0.38 & & & & \\
\hline 28 & 183339.32 & -182321.0 & 14.10 & -0.37 & & & & \\
\hline 29 & 182912.06 & -18445.8 & 13.06 & -0.37 & 800 & 800 & $\mathrm{AB}$ & - \\
\hline 30 & 183916.88 & -18118.0 & 13.20 & -0.36 & 4800 & 9300 & early-B & early-B \\
\hline 31 & 184125.28 & -175730.1 & 11.93 & -0.36 & & & & \\
\hline 32 & 182937.59 & -19736.4 & 12.93 & -0.35 & 740 & 370 & $\mathrm{AB}$ & PN? \\
\hline 33 & 182758.07 & -185740.9 & 11.34 & -0.34 & 2200 & 2000 & $\mathrm{AB}$ & - \\
\hline 34 & 183347.27 & -193248.2 & 15.05 & -0.33 & 3400 & 5000 & early-B & early B \\
\hline 35 & 184532.45 & -19023.2 & 14.80 & -0.33 & 0 & 0 & - & - \\
\hline 36 & 183856.46 & -193659.7 & 11.26 & -0.33 & 8500 & 9900 & mid-B & early-B \\
\hline 37 & 183416.24 & -173613.5 & 12.33 & -0.33 & & & & \\
\hline 38 & 183459.86 & -181918.7 & 13.28 & -0.33 & 16000 & 21000 & mid-B & late-B \\
\hline 39 & 183356.02 & -193819.6 & 12.68 & -0.33 & 34000 & 26000 & late-B & late-B \\
\hline 40 & 182952.31 & -191245.5 & 11.96 & -0.32 & & & & \\
\hline 41 & 184047.95 & -18562.3 & 15.44 & -0.32 & 130 & 480 & - & $\mathrm{AB} ?$ \\
\hline 42 & 182854.44 & -183922.5 & 12.95 & -0.32 & 1900 & 1000 & late-B? & $\mathrm{AB} ?$ \\
\hline 43 & 183711.53 & -203932.2 & 11.11 & -0.32 & 12000 & 3500 & late-B & $\mathrm{AB}$ \\
\hline 44 & 183938.53 & -21239.2 & 15.20 & -0.30 & 1100 & 1100 & early-B & $\mathrm{AB}$ \\
\hline 45 & 183423.25 & -215353.6 & 11.88 & -0.30 & 0 & 360 & - & - \\
\hline 46 & 18327.33 & -204327.0 & 14.25 & -0.30 & 160 & 540 & - & $\mathrm{AB} ?$ \\
\hline 47 & 183825.20 & -182944.3 & 12.45 & -0.30 & & & & \\
\hline 48 & 183728.39 & -185211.1 & 11.17 & -0.30 & 120 & 150 & - & - \\
\hline 49 & 184029.50 & -174043.8 & 12.27 & -0.30 & 6700 & 7800 & mid-B & mid-B \\
\hline 50 & 183354.09 & -201544.1 & 15.92 & -0.30 & & & & \\
\hline 51 & 183641.39 & -195242.8 & 11.23 & -0.29 & & & & \\
\hline 52 & 183128.92 & -185745.2 & 10.01 & -0.29 & & & & \\
\hline 53 & 183824.42 & -194439.8 & 14.55 & -0.28 & & & & \\
\hline 54 & 183040.89 & -18120.8 & 11.47 & -0.28 & 1900 & 1300 & early-B & $\mathrm{AB}$ \\
\hline 55 & 183026.04 & -18416.4 & 10.14 & -0.28 & & & & \\
\hline 56 & 18297.97 & -175535.6 & 12.88 & -0.28 & 2600 & 3000 & early-B & $\mathrm{AB} ?$ \\
\hline 57 & 18343.76 & -181533.7 & 10.66 & -0.27 & & & & \\
\hline 58 & 183720.09 & -173331.3 & 12.71 & -0.26 & 5900 & 6000 & late-B & early-B \\
\hline 59 & 184052.73 & -202024.4 & 12.65 & -0.26 & 1200 & 750 & $\mathrm{AB}$ & $\mathrm{AB}$ \\
\hline 60 & 182849.83 & -204756.9 & 12.20 & -0.26 & 850 & 320 & $\mathrm{AB}$ & - \\
\hline 61 & 182734.55 & -183433.3 & 11.63 & -0.25 & 480 & 900 & - & late? \\
\hline
\end{tabular}




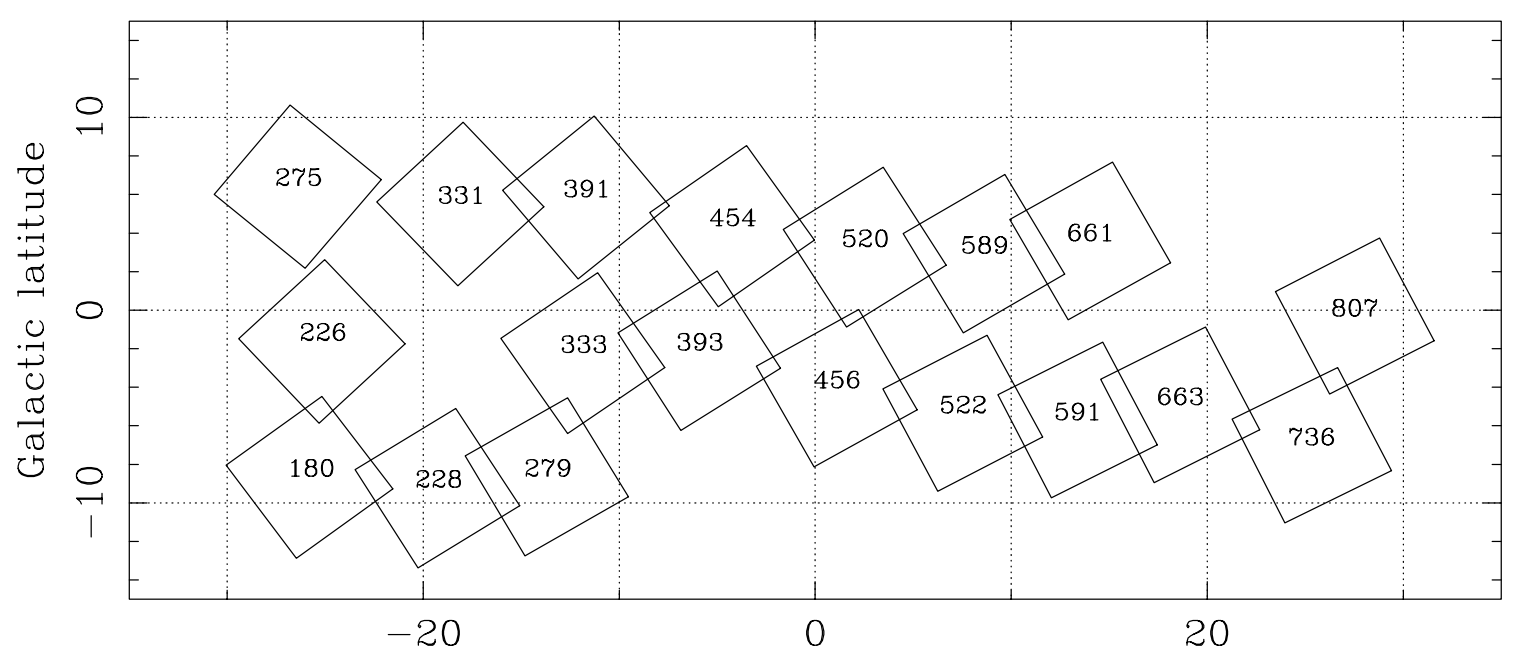

Galactic longitude

Fig. 1. The 19 Schmidt fields in the Galactic centre survey. Galactic longitude is with respect to the Galactic centre; field numbers are standard UKSTU field designations described in Tritton (1983).

Blue spectra:

O type: Presence of He II lines at 4200 and

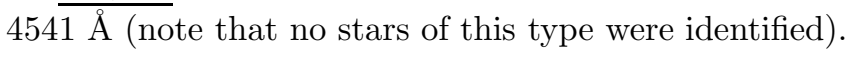

Early-B type: Relatively weak and narrow Balmer lines coupled with a well developed neutral helium spectrum. The Ca II line (at $3933 \AA$ ), Mg II close doublet (at $4481 \AA$ ) should be weak or absent (the interstellar components in the CaII lines should normally appear weak at this spectral resolution).

Mid-B type: Stronger and broader Balmer lines coupled with a weak but still distinct neutral helium spectrum. The CaII, Mg II, if present, are weak (with, for example, the He I line at $4471 \AA$ being stronger than Mg II).

Late-B type: Strong and broad Balmer lines coupled with a very weak neutral helium spectrum. Normally moderate Ca II, and Mg II features (with, for example, the Mg II doublet being at least as strong as the He I line at $4471 \AA$ ).

AB-type: Balmer lines (often strong and broad) coupled with no detectable neutral helium spectrum. Moderate CaII, and MgII features (if observable). Such stars will normally be late-B/early-A type for high quality spectra but might also be early or mid-B type for lower quality spectra (in this case they will usually be designated $\mathrm{AB}$ ).

Late-type: Strong CaII line with the $G$-band often present. No neutral helium lines visible.

Planetary Nebulae (PN): Balmer lines in emission with underlying continuum being weak. Often the neutral helium lines also observed in emission. Note that at the available spectral resolution, the candidates could also be faint post-asymptotic-giant-branch stars such as LSIV 12111 (Conlon et al. 1993).

Subdwarf (sd): Strong Balmer lines coupled with the absence of Mg II or Ca II lines expected in late-B/A-type stars.
Peculiar or composite: A small number of spectra of moderate or high quality could not be satisfactorily classified. Some of these appeared to be composite with both early-type and late-type spectral characteristics.

\section{Red spectra:}

Early-B-type: Relatively weak and narrow Balmer $\mathrm{H} \alpha$ line and detectable neutral helium lines at 5876 and $6678 \AA$. The Na I doublet at $5890 \AA$ (often blended with the nearby He I line) should be weak.

Mid-B-type: Stronger and broader $\mathrm{H} \alpha$ coupled with a weak but still distinct neutral helium spectrum. The NaI features should be weak.

AB type: $\mathrm{H} \alpha$ (often strong and broad) coupled with no detectable neutral helium spectrum. Normally the Na I doublet is weak. Such stars will usually be late-B/early-A type for the high quality spectra but might also be early or mid-B type for lower quality spectra (in this case they will normally be designated AB?).

Late-type: The Na I doublet should be strong with no neutral helium lines visible. The Balmer $\mathrm{H} \alpha$ line is often in emission.

Planetary Nebulae $(\mathrm{PN})$ : Balmer $\mathrm{H} \alpha$ line in emission with underlying continuum being weak. Often the neutral helium lines and the [N II] doublet at 6548 and $6583 \AA$ are also observed in emission. As for the blue spectra, these targets could be post-asymptotic-giant-branch stars.

The classification of the blue and red spectra were undertaken independently. Normally the assignments were consistent but in a few cases they disagreed. These spectra were checked to ensure that the discrepancy did not arise from a typographical error but otherwise the classifications remained unchanged. In all cases, more weight should be given to classification from the blue spectra, given both their higher spectral resolution and larger number of spectral diagnostics. Indeed the classifications from 
the red spectra are probably best considered as consistency checks of the classifications from the blue spectra. In particular the $\mathrm{H} \alpha$ line is useful for distinguishing between normal, massive B-type main sequence stars (where the line is in absorption) and evolved, hot PAGB stars where the line is often in emission (Conlon et al. 1993).

The classifications are summarised for the seven fields in Tables 2 to 8 . Also listed are the mean counts in the summed spectra for the wavelength ranges 3900 to $4600 \AA$ (blue) and 5600 to $6800 \AA$ (red), which as discussed by DSH should allow an estimate of the continuum signal-to-noise ratio. The quality of the spectra was generally higher than in DSH - this arises mainly from the better seeing conditions. The limits in the range of quality is reasonably well illustrated by Fig. 3 in DSH, although the quality of our best spectra is higher than shown there.

\subsection{Early-type candidates}

For the B-type candidates, an attempt has been made to obtain a more reliable classification and to estimate atmospheric parameters. Note that we excluded targets where the classification was uncertain or based solely on the red spectra. Equivalent widths (listed in Tables 9 to 11) were estimated, using the methods discussed by DSH, for the Balmer, $\mathrm{H} \delta, \mathrm{H} \gamma$, for the neutral helium lines at 4026, 4143, 4387 and $4471 \AA$ and the metal lines, MgII at $4481 \AA$ and CaII at $3933 \AA$. No attempt was made to measure features in the red spectra, due to the lower spectral resolution, and nebular emission contaminating the $\mathrm{H} \alpha$ line (even after correction using sky fibres) and the relative paucity of suitable features. For the late-B type sample, no neutral helium line equivalent widths are included as these features were either very weak or absent.

Assuming a normal helium to hydrogen ratio, the relative strength of the neutral helium and hydrogen lines can provide an estimate of the stellar effective temperature. Then the absolute strength of the hydrogen lines can be used to estimate the gravity. We have followed the procedures discussed in detail by DSH and based on a grid of models generated with the ATLAs9 code of Kurucz (1991) and an LTE model atmosphere code (Smartt et al. 1996b). The atmospheric parameters are again summarized in Tables 9 to 11 and include estimates for stars in the three fields discussed by DSH. We note the following:

- for stars with high effective temperatures the ratio of the strengths of the helium and hydrogen lines do not significantly vary with temperature. Hence for some of the stars in Table 9, we are only able to give a lower limit to the effective temperature $(\geq 20000 \mathrm{~K})$ and a corresponding lower limit to the gravity;

- the continuum for the hydrogen lines was arbitrarily defined at $\pm 16 \AA$ from the line centre to facilitate measurement. This leads to the theoretical equivalent widths becoming relatively insensitive to gravity when the lines are strong (i.e. at low effective temperatures and high gravities). Hence in these cases, we cannot constrain the gravity and these are designated hgl (for high gravity limit) in Table 11.

Even for the best observed stars, there will be considerable uncertainties in the atmospheric parameters. Realistic error estimates would be at least $\pm 1000-2000 \mathrm{~K}$ in effective temperature and $\pm 0.2-0.3$ in the logarithmic gravity. The uncertainties for the lower quality spectra could be larger.

Below we discuss the atmospheric parameters for each group of preliminary spectral-types. To simplify the discussion, stars are represented by two numbers (in square brackets) representing the field and the star:

Early-B-type candidates: DSH's list of 10 candidates has now increased to 56 targets, with 23 stars in the high effective temperature limit. In these cases only lower limits can be estimated for both the temperature and gravity. Assuming that these stars are main (or near) main sequence stars with logarithmic gravities less than 4.2 dex (Claret 1995), then the upper values of their effective temperatures can also be estimated. These would be less than $30000 \mathrm{~K}$ for all the stars, apart from $[279,19]$ and hence consistent with the lack of an observed He II spectrum (see DSH for details). The target, [279,19], would appear to be either an evolved giant or supergiant (and hence a very distant object) or, more likely, a highly evolved postasymptotic-giant branch or post-horizontal-branch star.

The spectra of some targets in the high temperature limit are of sufficient quality that metal lines can be seen. Those for the six stars with the most prominent metal lines are illustrated in Fig. 2. The spectra of five stars show evidence of lines due to O II (at 4070-4090 $\AA$ and near to $\mathrm{H} \gamma$ ) and in some cases Si III (at 4550-4575 $\AA$ ). Assuming that the metal abundances are relatively normal, this would imply a spectral type of B0 or B1. The sixth star, $[589,53]$, is probably cooler as the O II spectrum is not seen, while the $\mathrm{C}$ II at $4267 \AA$ is present; in this case a spectral type of B2 or later would appear to be appropriate.

There are nine stars that have helium to hydrogen line ratios that imply effective temperatures of 17000 to $18000 \mathrm{~K}$ but as discussed by DSH, their observational uncertainties are consistent with them being in the high temperature limit. However five targets, if they are main sequence stars, have effective temperatures less than approximately $20000 \mathrm{~K}$ as otherwise they would have too large a surface gravity. For the other four targets ([333,60], $[391,23],[520,15],[591,34])$, their hydrogen line strengths are weaker and hence would be consistent with a higher effective temperature main sequence star. The spectra of $[520,15]$ is of too low a quality to distinguish metal lines but those of the other three targets are shown in Fig. 3. For two stars $([333,60],[591,34])$, the O II spectrum appears to be present, which implies that their effective temperatures may indeed be greater than 20000 K. For [391,23], the absence of $\mathrm{O}$ II lines coupled with a prominent $\mathrm{C}$ II feature at $4267 \AA$ is compatible with its estimated effective temperature. 
Table 9. Equivalent widths for the early-B type stellar candidates and their estimated atmospheric parameters. Also listed are the candidates for fields 393, 454 and 456, which have been taken from DSH.

\begin{tabular}{|c|c|c|c|c|c|c|c|c|c|c|c|}
\hline Field & Star & 4101 & 4340 & 4026 & 4143 & 4387 & 4471 & 4481 & 3933 & $T_{\text {eff }}$ & $\log g$ \\
\hline 279 & 02 & 5.4 & 4.7 & 1.6 & 1.2 & $0.7:$ & 1.5 & 0.2 : & - & $\geq 20000$ & $\geq 4.2$ \\
\hline 279 & 04 & 4.6 & 5.3 & 2.3 & 1.7 & 1.0 & 1.8 & 0.3: & - & $\geq 20000$ & $\geq 4.2$ \\
\hline 279 & 05 & 4.7 & 4.3 & 1.5: & 0.8 & 1.4 & 1.3 & - & $0.3:$ & $\geq 20000$ & $\geq 3.9$ \\
\hline 279 & 07 & 3.9 & 4.0 & 2.0 & 1.0 & 1.0 & 1.4 & - & $0.3:$ & $\geq 20000$ & $\geq 3.6$ \\
\hline 279 & 08 & 5.5 & 5.7 & 2.1 & 1.1 & 1.0 & 2.1 & $0.2:$ & - & $\geq 20000$ & $\geq 4.5$ \\
\hline 279 & 09 & 3.6 & 4.2 & 1.2 & 0.8 & 0.8 & 1.3 & $\mathrm{p} ?$ & 0.5 : & $\geq 20000$ & $\geq 3.5$ \\
\hline 279 & 19 & 2.1 & 2.4 & 0.7 & 0.5 & 0.8 & 0.7 & - & $0.5:$ & $\geq 20000$ & $\geq 2.7$ \\
\hline 279 & 23 & 6.0 & 5.7 & 0.9 : & 0.9: & 0.5 : & 0.9 : & 0.2 : & 0.6 : & 16 000: & 4.1: \\
\hline 279 & 28 & 6.9 & 5.8 & 0.8: & - & 0.8: & 0.7 : & - & - & 15 000: & 4.2: \\
\hline 333 & 02 & 3.3 & 3.4 & $0.8:$ & - & - & 0.9 : & - & - & 16000 & 2.7 \\
\hline 333 & 11 & 5.3 & 5.6 & 0.9 & 0.5 & 0.5 : & 1.1 & 0.2 : & 0.4 & 15000 & 3.8 \\
\hline 333 & 43 & 5.7 & 5.7 & 0.8 & 0.5 : & 0.5 & 0.9 & 0.2 : & 0.3 & 14000 & 3.9 \\
\hline 333 & 49 & 6.0 & 6.0 & 0.9 & 0.5 : & 0.6 & 0.6 : & - & 0.5 & 14000 & 3.8 \\
\hline 333 & 60 & 4.2 & 4.6 & 1.2 & 0.8 & 0.9 & 1.3 & 0.2 : & 0.4 & 17000 & 3.4 \\
\hline 391 & 04 & 3.3: & 3.4: & 0.9 : & 0.6 : & 0.5 & $0.8:$ & - & 0.7 & $\geq 20000$ & $\geq 3.3$ \\
\hline 391 & 08 & 6.2 : & $6.7:$ & 1.7: & 1.0: & - & 1.8: & - & - & 18000 & 4.5 \\
\hline 391 & 09 & 2.9: & 3.6 & 0.9 & 0.4 : & 0.4 : & 1.1 & 0.2 & 0.6 & $\geq 20000$ & $\geq 3.2$ \\
\hline 391 & 11 & 5.6 & 5.7 & 1.2 & 0.5 : & 0.7 & 1.2 & 0.3 & 0.5 & 16000 & 3.8 \\
\hline 391 & 13 & 3.8 & 3.7 & 0.7 & 0.4 : & 0.5 : & 0.6 : & 0.2 & 0.6 & 16000 & 3.0 \\
\hline 391 & 16 & 5.8 & 5.8 & 1.5 & 0.7 & 0.7 & 1.2 & 0.3 & 0.3 & 17000 & 4.2 \\
\hline 391 & 23 & 4.1 & 4.5 & 1.1 & 0.7 & 0.7 & 0.9 & 0.3 & 0.7 & 17000 & 3.3 \\
\hline 391 & 57 & 6.4 & 6.5 & 1.0 & 0.3 : & 0.5 : & 0.8 & $0.3:$ & 0.3 & 14000 & 3.9 \\
\hline 393 & 3 & 3.6: & 2.5: & 1.3: & 1.5: & 1.0: & 1.0: & - & - & $\geq 20000$ & $\geq 3.2$ \\
\hline 393 & 13 & 3.8 & 3.5 & 1.5: & $0.8:$ & $0.8:$ & 0.9 : & - & - & $\geq 20000$ & $\geq 3.4$ \\
\hline 393 & 32 & 4.5 & 4.0 & 1.3: & 1.0 & 1.0 & 1.2 & 0.2 : & 0.5 : & $\geq 20000$ & $\geq 3.7$ \\
\hline 393 & 55 & 4.6 & 4.2 & - & - & - & 1.6 & - & - & $\overline{\geq} 20000$ & $\geq 3.8:$ \\
\hline 454 & 10 & 4.0: & 3.7 & 1.1 & 0.7 & 0.8 & 1.1 & - & 0.5 & $\geq 20000$ & $\geq 3.5$ \\
\hline 454 & 27 & 3.4: & 4.0 & 0.9 : & - & $\mathrm{p}$ & 1.4: & - & - & $\geq 20000:$ & $\geq 3.5:$ \\
\hline 456 & 5 & 4.1: & 3.8: & 1.5: & $\mathrm{p}$ & 1.0: & 1.6: & $\mathrm{p} ?$ & $\mathrm{p} ?$ & $\geq 20000$ & $\geq 3.5$ \\
\hline 456 & 18 & 5.6: & 4.4: & - & 0.6 : & $0.8:$ & 0.9 : & $0.3:$ & - & 18 000: & 4.0: \\
\hline 456 & 30 & 5.3: & 4.4: & - & - & 0.9 : & - & - & $\mathrm{p} ?$ & $18000:$ & 4.0: \\
\hline 456 & 39 & 3.4 & 3.1 & $0.8:$ & $\mathrm{p}$ & 0.7 & 1.2 & $\mathrm{p}$ & 0.7: & $\geq 20000$ & $\geq 3.3$ \\
\hline 520 & 01 & 3.4 & $3.2:$ & 0.5 : & - & - & 0.7 : & - & - & $14000:$ & $2.5:$ \\
\hline 520 & 05 & 5.3 & 4.5 & 0.8: & - & 0.8: & 1.4 & - & - & 15000 & 3.3 \\
\hline 520 & 10 & 3.7 & 3.4 & 1.2 & $0.7:$ & 0.9 & 1.0: & - & - & $\geq 20000$ & $\geq 3.4$ \\
\hline 520 & 15 & 4.0: & 4.2 & 1.0: & - & - & 0.6 : & - & 0.4 : & 17 000: & 3.3: \\
\hline 520 & 53 & 6.1 & 6.2 & 1.6: & - & 0.7 : & 1.1: & - & - & $16000:$ & 4.3: \\
\hline 522 & 11 & 5.7 & 5.5 & 1.4 & 0.6 & 0.8 & 1.3 & 0.2 : & 0.2 : & 18000 & 4.2 \\
\hline 522 & 15 & 3.8: & 4.5 & 1.3: & - & $0.8:$ & $\mathrm{p}$ & - & - & $\geq 20000$ & $\geq 3.7$ \\
\hline 522 & 16 & 5.4 & 5.2 & 1.1: & 0.7 : & - & 0.9 : & 0.4 : & - & $16000:$ & 3.7: \\
\hline 522 & 23 & 6.7 & 6.7 & 0.9 & 0.4 & 0.6 & 1.0 & 0.3: & $0.4:$ & 15000 & 4.3 \\
\hline 522 & 43 & 5.4 & 5.6 & 1.0 & - & - & 1.2: & - & 0.4: & 15000 & 3.7 \\
\hline 522 & 56 & 6.0 & 6.2 & 1.1 & 0.4 : & 0.5 : & 0.8 & $0.3:$ & - & 15000 & 4.0 \\
\hline 589 & 33 & 3.5 & 4.2 & 1.1 & 0.6 : & $0.7:$ & 1.4 & - & - & $\geq 20000$ & $\geq 3.5$ \\
\hline 589 & 43 & 6.2 & 5.7 & 1.2 & 0.6 : & - & 0.9 & $\mathrm{p} ?$ & - & 16000 & 4.1 \\
\hline 589 & 53 & 4.6 & 5.1 & 1.4 & 0.7 & 0.8 & 1.3 & - & $0.3:$ & $\geq 20000$ & $\geq 4.1$ \\
\hline 589 & 61 & 7.1: & 6.4: & 1.4: & - & - & - & - & - & 16 000: & 4.5: \\
\hline 591 & 05 & 3.8 & 3.7 & 1.0 & 0.4 : & 0.5 : & 1.1 & $0.2:$ & 0.6 : & $\geq 20000$ & $\geq 3.5$ \\
\hline 591 & 08 & 5.1 & 5.0 & 1.2 & 0.6 & 0.8 & 1.3 & 0.2 : & $0.5:$ & 16000 & 3.7 \\
\hline 591 & 20 & 5.9 & 5.4 & $0.9:$ & - & 0.7 & 0.7 : & - & $0.4:$ & 15 000: & 3.7: \\
\hline 591 & 25 & 5.6 & 5.5 & 1.1 & 0.5 & 0.7 & 1.0 & 0.2 : & $0.3:$ & 16000 & 3.9 \\
\hline 591 & 30 & 4.0 & 4.2 & 1.3: & 0.7 : & 0.9 & 1.3 & $0.3:$ & 0.5 : & $\geq 20000$ & $\geq 3.6$ \\
\hline 591 & 34 & 3.8 & 3.9 & 1.0: & 0.6 : & $0.7:$ & 0.6 : & - & $0.7:$ & 18 000: & 3.3: \\
\hline 591 & 44 & 6.9: & 6.4: & 1.1: & - & 0.6 : & $0.9:$ & 0.3: & $0.5:$ & $14000:$ & 4.0: \\
\hline 591 & 54 & 6.0 & 5.6 & 0.9 & 0.4 : & 0.6 : & 1.0 & 0.4: & - & 15000 & 3.8 \\
\hline 591 & 56 & 5.6: & 5.7 & 0.9 : & - & $0.5:$ & 0.9 : & - & - & 15 000: & 3.7: \\
\hline
\end{tabular}


Table 10. Equivalent widths for the mid-B type stellar candidates and their estimated atmospheric parameters. Also listed are the candidates for fields 393, 454 and 456, which have been taken from DSH.

\begin{tabular}{|c|c|c|c|c|c|c|c|c|c|c|c|}
\hline Field & Star & 4101 & 4340 & 4026 & 4143 & 4387 & 4471 & 4481 & 3933 & $T_{\text {eff }}$ & $\log g$ \\
\hline 279 & 18 & 6.9 & 7.0 & 0.8 & $0.2:$ & - & 0.7 : & $p$ & $0.3:$ & 13000 & 4.0 \\
\hline 279 & 20 & 7.6 & 7.2 & 0.7: & - & 0.3: & 0.9: & - & 0.9 & 13000 & 4.1 \\
\hline 279 & 22 & 4.3 & 4.1 & $0.3:$ & - & - & $0.5:$ & - & $\mathrm{p}$ & 12000 & 2.5 \\
\hline 279 & 34 & 8.0 & 7.0 & 0.7 : & 0.3: & - & 0.9: & 0.5 : & 0.6 : & 13000 & 4.2 \\
\hline 279 & 38 & 7.0 & 6.6 & $0.3:$ & - & - & $0.3:$ & 0.2 : & $0.4:$ & 11000 & 3.1 \\
\hline 279 & 42 & 8.1 & 8.5 & 0.9 : & - & 0.3: & $0.6:$ & $0.4:$ & $0.5:$ & 13000 & 4.5 \\
\hline 279 & 49 & 8.8 & 8.2 & 0.5 : & - & - & 0.5 : & 0.4 : & 0.4: & 12000 & 4.5 \\
\hline 279 & 51 & 7.7 & 7.7 & 0.7 & $0.4:$ & 0.4: & 1.0: & 0.2 : & 0.6 : & 13000 & 4.3 \\
\hline 333 & 04 & 6.7: & 6.7 & 0.9: & 0.4 : & $0.5:$ & 1.1: & 0.2 : & 0.4 : & 14000 & 4.1 \\
\hline 333 & 17 & 7.6 & 8.2 & 0.3: & - & - & $0.3:$ & $0.3:$ & 0.4: & 12 000: & 4.0: \\
\hline 333 & 19 & 6.5 & 6.2 & 0.6 & - & 0.4: & 0.5 : & 0.2 : & $0.5:$ & 13000 & 3.6 \\
\hline 333 & 23 & 7.4 & 7.4 & 0.6 & $0.2:$ & 0.4: & 0.6 & 0.4: & $0.5:$ & 13000 & 3.8 \\
\hline 333 & 33 & 7.3 & 7.4 & 0.5 : & $0.2:$ & 0.3: & 0.6 & $0.3:$ & 0.5 : & 12000 & 3.7 \\
\hline 333 & 34 & 6.7 & 7.0 & 0.4 : & - & $0.3:$ & 0.6 : & $0.4:$ & $0.4:$ & 12000 & 3.6 \\
\hline 333 & 48 & 7.9 & 7.8 & $0.4:$ & - & - & $0.4:$ & $0.3:$ & $0.4:$ & 11000 & 3.5 \\
\hline 333 & 56 & 6.0 & 5.9 & 0.5 & - & 0.3: & 0.4 : & 0.2 : & 0.2 : & 13000 & 3.5 \\
\hline 333 & 57 & 7.6 & 7.3 & 0.5 : & $0.2:$ & 0.3: & 0.4 : & $0.3:$ & 0.3: & 12000 & 3.7 \\
\hline 333 & 61 & 6.7 & 6.3 & 0.8: & $0.5:$ & 0.6: & 0.6 & 0.4 : & 0.4: & 14000 & 3.9 \\
\hline 391 & 15 & 6.6 & 6.5 & 0.6: & - & - & 0.4 : & 0.4: & 0.4: & 13000 & 3.7 \\
\hline 391 & 18 & 6.8 & 6.9 & $0.5:$ & - & - & 0.6 & 0.2 : & 0.7: & 13000 & 4.0 \\
\hline 391 & 63 & 7.4 & 7.6 & 0.5 : & - & - & $0.3:$ & 0.2 : & $0.3:$ & 12000 & 3.8 \\
\hline 393 & 24 & $\mathrm{p}$ & 5.0 & $\mathrm{p}$ & $\mathrm{p}$ & - & - & $\mathrm{p} ?$ & - & $\leq 15000$ & $\leq 3.5:$ \\
\hline 393 & 25 & 6.9 & 6.7 & 0.5: & $\mathrm{p} ?$ & 0.3: & 0.3: & $\mathrm{p} ?$ & - & 12000 & 3.6 \\
\hline 393 & 27 & 7.3 & 7.4 & 0.5 : & $\mathrm{p} ?$ & $0.2:$ & $0.3:$ & $0.3:$ & $0.3:$ & 11000 & 3.4 \\
\hline 393 & 41 & 5.2 & 5.3 & - & $\mathrm{p} ?$ & 0.3: & 0.5 : & 0.2 : & 0.2 : & 12000 & 3.0 \\
\hline 393 & 45 & 9.1 & 9.0 & $\mathrm{p} ?$ & $0.4:$ & - & 0.5 : & 0.4 : & 1.1 & 12000 & 4.0 \\
\hline 393 & 53 & 7.0 & 6.9 & 0.9 & $0.4:$ & $0.5:$ & 0.8 & 0.3 & $0.4:$ & 14000 & 4.3 \\
\hline 454 & 13 & 6.3 & 5.9 & 1.0 & $0.5:$ & 0.6 & 0.9 & 0.2 & $0.3:$ & 15000 & 4.0 \\
\hline 454 & 14 & 5.2 & 4.8 & 0.9 & 0.4: & 0.6 & 0.9 & 0.2 & 0.2 & 15000 & 3.5 \\
\hline 454 & 16 & 6.8 & 6.8 & $0.3:$ & $\mathrm{p}$ & 0.2 & 0.2 & 0.2 & 0.2 & 11000 & 3.2 \\
\hline 454 & 17 & 7.5 & 7.2 & 0.3 & $\mathrm{p}$ & 0.2 & 0.3 & 0.2 & 0.3 & 11000 & 3.4 \\
\hline 454 & 24 & 7.3 & 7.0 & 0.2 & 0.1: & - & 0.3 & 0.2 & 0.3 & 11000 & 3.3 \\
\hline 454 & 36 & 6.7 & 6.5 & 0.9 & 0.4 & 0.4 & 0.8 & 0.2 & 0.2 & 14000 & 4.0 \\
\hline 454 & 38 & 7.5 & 7.4 & 0.3: & 0.3 & 0.2 & 0.5 & 0.3 & 0.3 & 12000 & 3.8 \\
\hline 456 & 16 & 6.4 & 6.1 & 0.4 : & $\mathrm{p}$ & 0.3: & 0.3: & 0.2 & 0.3 & 12000 & 3.3 \\
\hline 456 & 25 & 5.0 & 4.9 & $\mathrm{p}$ & - & $0.5:$ & $0.5:$ & - & 0.8: & 15000 & 3.5 \\
\hline 456 & 33 & 6.7 & 6.0 & 1.1: & 0.4: & - & 0.4: & 0.4 : & - & 15000 & 4.2 \\
\hline 456 & 37 & 6.1 & 5.7 & 0.7: & $0.5:$ & 0.7: & $0.6:$ & $0.3:$ & 0.2 : & 16000 & 4.1 \\
\hline 456 & 43 & 7.8 & 6.8 & 0.2 : & - & 0.2 : & 0.3: & 0.2 : & $0.3:$ & 11000 & 3.4 \\
\hline 456 & 53 & 6.9 & 6.8 & 0.3 & - & $\mathrm{p} ?$ & 0.3: & 0.2 : & 0.2 : & 11000 & 3.1 \\
\hline 522 & 10 & 6.9 & 6.7 & 0.8 & 0.3: & 0.5: & 0.6: & 0.3 : & $0.5:$ & 14 000: & 4.1: \\
\hline 522 & 21 & 8.7 & 8.6 & 0.4: & - & - & - & - & - & 12 000: & 4.4: \\
\hline 522 & 27 & 6.5: & 7.3 & 0.8: & - & - & - & - & - & 14 000: & 4.1: \\
\hline 522 & 50 & 7.9 & 7.4 & 0.7 & - & - & - & - & - & 13 000: & 4.3: \\
\hline 522 & 52 & 7.0 & 7.1 & $0.4:$ & - & $0.2:$ & $0.3:$ & 0.2 : & $0.3:$ & 12000 & 3.6 \\
\hline 589 & 06 & 6.4 & 6.0 & $0.8:$ & $0.4:$ & 0.3: & 0.7: & $0.3:$ & 0.4 : & 14000 & 3.8 \\
\hline 589 & 31 & 7.5 & 7.4 & 0.4: & - & - & 0.4 : & - & 0.6: & 12 000: & 3.8: \\
\hline 589 & 54 & 8.1 & 7.9 & 0.3: & - & - & $0.3:$ & 0.2 : & $0.5:$ & $11000:$ & 3.6: \\
\hline 591 & 12 & 6.9 & 7.1 & $0.6:$ & 0.3: & $0.4:$ & $0.6:$ & $0.3:$ & 0.4: & 13000 & 3.9 \\
\hline 591 & 18 & 7.9 & 7.8 & 0.3: & - & 0.2 : & - & - & $0.5:$ & $11000:$ & 3.5 \\
\hline 591 & 24 & 6.6 & 6.9 & $0.4:$ & - & 0.3: & 0.5 : & 0.3 : & 0.6: & 12000 & 3.5 \\
\hline 591 & 36 & 6.3 & 6.5 & 0.8 & 0.3: & 0.4: & 0.7 & 0.2 : & $0.2:$ & 14000 & 3.9 \\
\hline 591 & 38 & 7.4 & 7.3 & $0.3:$ & - & $0.3:$ & - & - & 0.6 : & $11000:$ & 3.4: \\
\hline 591 & 49 & 7.8 & 7.9 & 0.5: & - & - & $0.7:$ & $0.3:$ & 0.4: & 12000 & 4.0 \\
\hline
\end{tabular}

The remaining targets have effective temperature estimates between 14000 and $16000 \mathrm{~K}$, which are low for our original spectral classification as early-B type. We note that all these targets are from our current spectroscopy, where the data quality is generally significantly higher than that discussed by DSH. Hence, even relatively 
Table 11. Equivalent widths for the late-B type stellar candidates and their estimated atmospheric parameters. Also listed are the candidates for fields 393, 454 and 456, which have been taken from DSH.

\begin{tabular}{|c|c|c|c|c|c|c|c|}
\hline Field & Star & 4101 & 4340 & 4481 & 3933 & $T_{\text {eff }}$ & $\log g$ \\
\hline 279 & 10 & 4.4 & 4.6 & - & 0.7 : & $\leq 13000$ & $\leq 2.8$ \\
\hline 279 & 39 & 8.6 & 8.4 & $0.3:$ & 0.4 : & $\leq 12000$ & $\leq 4.5$ \\
\hline 279 & 46 & 9.9 & 9.1 & $0.3:$ & 0.5 : & $\leq 12000$ & hgl \\
\hline 279 & 55 & 8.4 & 8.1 & $0.2:$ & 0.4 : & $\leq 12000$ & $\leq 4.3$ \\
\hline 279 & 57 & 9.7 & 9.1 & $0.3:$ & $0.3:$ & $\leq 12000$ & hgl \\
\hline 279 & 58 & 8.5 & 8.5 & 0.4: & $0.8:$ & $\leq 12000$ & $\leq 4.5$ \\
\hline 333 & 12 & 9.9 & 9.8 & $0.3:$ & $0.8:$ & $\leq 12000$ & hgl \\
\hline 333 & 14 & 9.0 & 8.3 & $0.4:$ & 0.4 : & $\leq 12000$ & $\leq 4.7$ \\
\hline 333 & 15 & 6.6 & 6.3 & - & $0.3:$ & $\leq 12000$ & $\leq 3.4$ \\
\hline 333 & 18 & 9.8 & 9.9 & $0.4:$ & 0.6 : & $\leq 12000$ & hgl \\
\hline 333 & 22 & 9.3 & 9.0 & $0.6:$ & 0.6 : & $\leq 12000$ & hgl \\
\hline 333 & 24 & 8.8 & 8.5 & $0.3:$ & 0.6 : & $\leq 12000$ & $\leq 4.6$ \\
\hline 333 & 26 & 8.7 & 8.3 & $0.3:$ & 0.6 : & $\leq 12000$ & $\leq 4.5$ \\
\hline 333 & 44 & 9.8 & 9.2 & $0.3:$ & $0.4:$ & $\leq 12000$ & hgl \\
\hline 333 & 55 & 6.6 & 6.2 & - & 0.4 : & $\leq 12000$ & $\leq 3.4$ \\
\hline 391 & 10 & 7.7 & 7.6 & $0.4:$ & 0.6 : & $\leq 12000$ & $\leq 4.0$ \\
\hline 391 & 12 & 6.9 & 6.8 & $0.3:$ & 0.4 : & $\leq 12000$ & $\leq 3.6$ \\
\hline 391 & 21 & 8.7 & 8.3 & $0.4:$ & 0.6 : & $\leq 12000$ & $\leq 4.5$ \\
\hline 391 & 26 & 9.4 & 8.9 & $0.3:$ & 0.6 : & $\leq 12000$ & $\overline{\mathrm{hgl}}$ \\
\hline 391 & 34 & 9.4 & 8.8 & $0.5:$ & $0.8:$ & $\leq 12000$ & hgl \\
\hline 391 & 38 & 9.4 & 8.9 & $0.5:$ & 0.6 : & $\leq 12000$ & hgl \\
\hline 391 & 39 & 9.8 & 9.4 & $0.4:$ & 0.7 : & $\leq 12000$ & hgl \\
\hline 391 & 44 & 9.8 & 9.1 & $0.5:$ & 0.5 : & $\leq 12000$ & hgl \\
\hline 391 & 49 & 8.0 & 8.2 & $0.3:$ & 0.4: & $\leq 12000$ & $\leq 4.2$ \\
\hline 391 & 64 & 9.1 & 9.2 & $0.3:$ & $0.7:$ & $\leq 12000$ & hgl \\
\hline 393 & 1 & 4.4 & 4.3 & - & - & $\leq 13000$ & $\leq 2.7$ \\
\hline 393 & 28 & 8.5 & 8.5 & $0.3:$ & 0.7 & $\leq 12000$ & $\leq 4.5$ \\
\hline 454 & 11 & 8.8 & 8.4 & $0.3:$ & 0.2 & $\leq 12000$ & $\leq 4.5$ \\
\hline 456 & 36 & 6.0 & 5.0 & $0.5:$ & 0.8: & $\leq 13000$ & $\leq 3.0$ \\
\hline 456 & 45 & 8.6 & 8.1 & $0.3:$ & 1.0 & $\leq 12000$ & $\leq 4.4$ \\
\hline 456 & 51 & 9.5 & 8.6 & $0.4:$ & 0.7 & $\leq 12000$ & hgl \\
\hline 454 & 58 & 8.9 & 8.7 & $0.4:$ & 0.4 & $\leq 12000$ & $\leq 4.8$ \\
\hline 522 & 18 & 9.5 & 9.1 & - & $0.5:$ & $\leq 12000$ & hgl \\
\hline 522 & 42 & 9.6 & 9.0 & 0.4 : & $0.8:$ & $\leq 12000$ & hgl \\
\hline 589 & 4 & 4.7 & 5.2 & - & - & $\overline{\leq} 13000$ & $\leq 2.8$ \\
\hline 589 & 10 & 7.4 & 7.3 & - & 0.4 : & $\leq 12000$ & $\leq 3.8$ \\
\hline 589 & 13 & 9.3 & 8.8 & - & $0.3:$ & $\leq 12000$ & $\overline{\mathrm{hgl}}$ \\
\hline 589 & 15 & 7.6 & 7.4 & $0.2:$ & 0.4 : & $\leq 12000$ & $\leq 3.9$ \\
\hline 589 & 19 & 8.3 & 7.7 & $0.3:$ & 0.5 : & $\leq 12000$ & $\leq 4.2$ \\
\hline 589 & 21 & 9.1 & 9.6 & $0.6:$ & $0.8:$ & $\leq 12000$ & hgl \\
\hline 589 & 23 & 8.9 & 8.9 & $0.4:$ & 0.4 : & $\leq 12000$ & $\leq 4.9$ \\
\hline 589 & 25 & 8.5 & 8.3 & $0.4:$ & 0.6 : & $\leq 12000$ & $\leq 4.4$ \\
\hline 589 & 35 & 9.8 & 9.5 & $0.5:$ & - & $\leq 12000$ & hgl \\
\hline 589 & 40 & 9.6 & 9.9 & $0.4:$ & $0.7:$ & $\leq 12000$ & hgl \\
\hline 589 & 44 & 9.0 & 9.2 & $0.3:$ & - & $\leq 12000$ & hgl \\
\hline 589 & 45 & 6.2 & 6.6 & - & - & $\leq 12000$ & $\leq 3.4$ \\
\hline 589 & 51 & 9.3 & 9.0 & $0.4:$ & 0.4 : & $\leq 12000$ & hgl \\
\hline 591 & 39 & 9.3 & 9.6 & $0.4:$ & $0.8:$ & $\leq 12000$ & hgl \\
\hline 591 & 43 & 6.4 & 6.6 & - & $0.3:$ & $\leq 12000$ & $\leq 3.4$ \\
\hline 591 & 58 & 6.6 & 6.8 & $0.3:$ & $0.8:$ & $\leq 12000$ & $\leq 3.5$ \\
\hline
\end{tabular}

weak neutral helium lines are clearly visible in the current dataset and this may explain why they have been subjectively classified as early-B type.

For targets with specific effective temperature estimates, thirty three stars appear to be on (or near) the

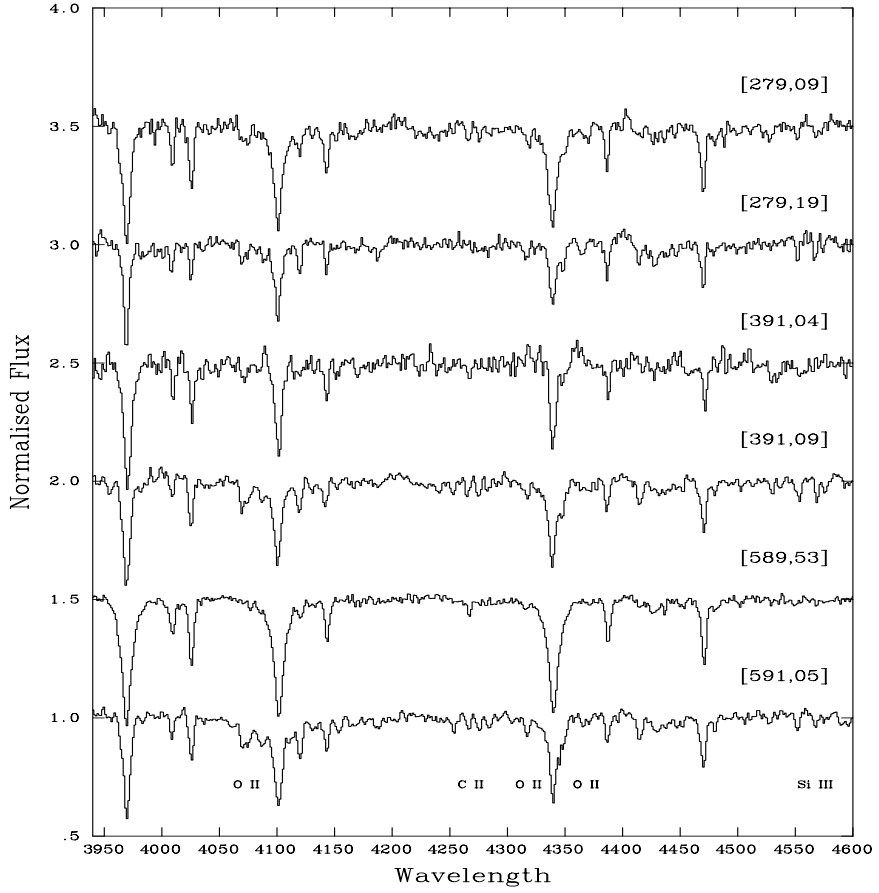

Fig. 2. Normalised spectra for six targets in the high effective temperature limit, with sufficient signal-to-noise to identify metal lines.

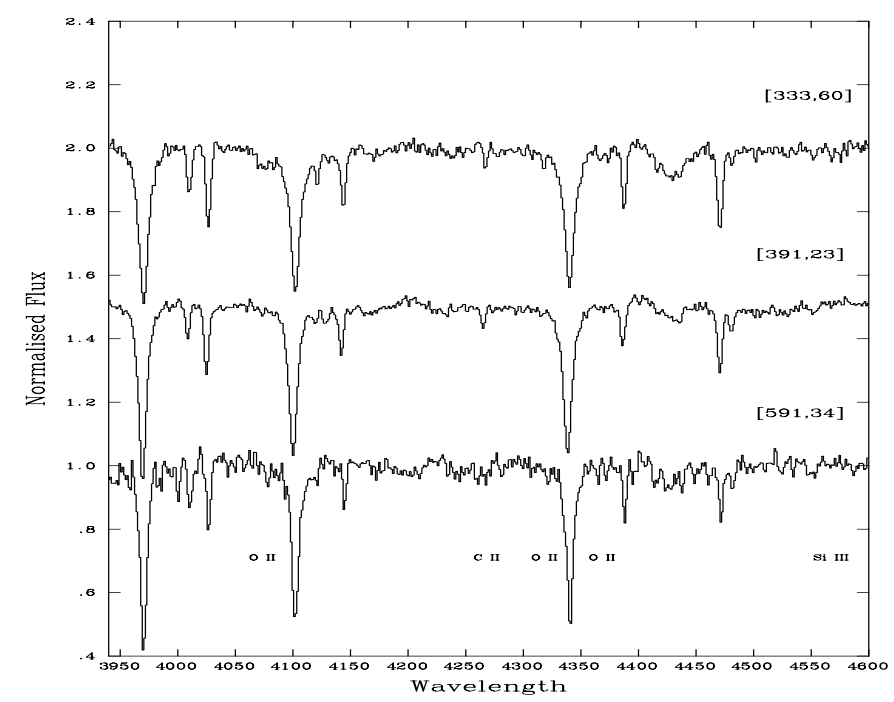

Fig. 3. Normalised spectra for three targets with effective temperature estimates of 17000 to $18000 \mathrm{~K}$, with sufficient signalto-noise to identify metal lines.

main sequence (with $\log g \geq 3.7$ ); however eight stars have lower gravities. The latter are likely to be a mixture of relatively young giants and old evolved stars. As discussed by, for example, Conlon et al. (1993), some evolved stars show strong emission in the Balmer $\mathrm{H} \alpha$ line. Seven of the lower gravity stars show no evidence of emission, while one $([391,13]])$ shows modest emission in the stellar core.

Mid-B-type candidates: Atmospheric parameters could be estimated for all the stars (apart from [393,24] discussed by DSH). Although the He I line strengths are often relatively poorly determined, they vary rapidly 
with the effective temperature. Hence the estimates of the atmospheric parameters should normally be reliable.

Our effective temperature estimates range from 11000 to $16000 \mathrm{~K}$ and indicate that our selection criteria have led to a homogeneous sample. The presence of cooler stars (which would be better classified as late-B type) reflects the difficulty of discriminating by eye between a range of weak neutral helium line strengths particularly when considering spectra of different quality (see the comment above on early-B type classification). As for the early-B type candidates, most of the stars appear to be on (or near) the main sequence (again with $\log g \geq 3.7$ ), but now a significant minority (23 stars out of a sample of 54) have lower gravities. Only sixteen of these targets have red spectra as DSH did not observe this spectral region for the F393 and F454 fields. Eleven targets show no evidence of emission, while four targets ([333,34], [333,48], [456,15], $[589,23])$ show modest emission in the stellar core. For one star $([333,19])$, the emission is very strong and almost certainly present in the stellar spectrum, implying that this star may be evolved. We stress that $\mathrm{H} \alpha$ emission is not present in the spectra of all post-AGB stars and hence we are unable to definitively classify the remaining low gravity targets.

Late-B/A-type candidates: For all these stars the neutral helium lines were either absent or too weak to measure. We have therefore assumed that they have equivalent widths of less than $0.5 \AA$. This should be appropriate for the spectra with low counts, but will be too conservative for the better quality data. For example, the metal line equivalent widths listed in Tables 9 to 11 are as small at $0.2 \AA$. However, as discussed by DSH, it was not felt that the data warranted assigning different equivalent width limits to each spectrum as these would, in any case, also have been wavelength dependent. The limits on the atmospheric parameters estimates are given in Table 11. Note that as discussed above "hgl" implies that the hydrogen lines were too strong for a reliable upper limit to the gravity to be estimated. If a more stringent He I line equivalent width limit of $0.2 \AA$ was adopted, the upper limits on the effective temperature and logarithmic gravity would be reduced by typically $2000 \mathrm{~K}$ and 0.2 dex respectively.

The stars appear to fall into two subgroups. Forty targets have maximum gravities that are consistent with them being main sequence targets, whilst the other ten have lower gravities. As for the previous groups, these may either be relatively young stars evolving from the hydrogen main sequence or older post-AGB objects and indeed four stars $([279,10],[333,55],[391,12],[589,45])$ show moderate amounts of $\mathrm{H} \alpha$ emission.

\section{Discussion}

For the ten fields surveyed, we have identified 32 B-type stars with effective temperature, $T_{\text {eff }} \geq 17000 \mathrm{~K}$ and 78 targets with $11000 \leq T_{\text {eff }} \leq 16000 \mathrm{~K}$. Additionally 50 targets have effective temperatures $T_{\text {eff }} \leq 12000 \mathrm{~K}$ and are probably either late-B or early-A spectral type.

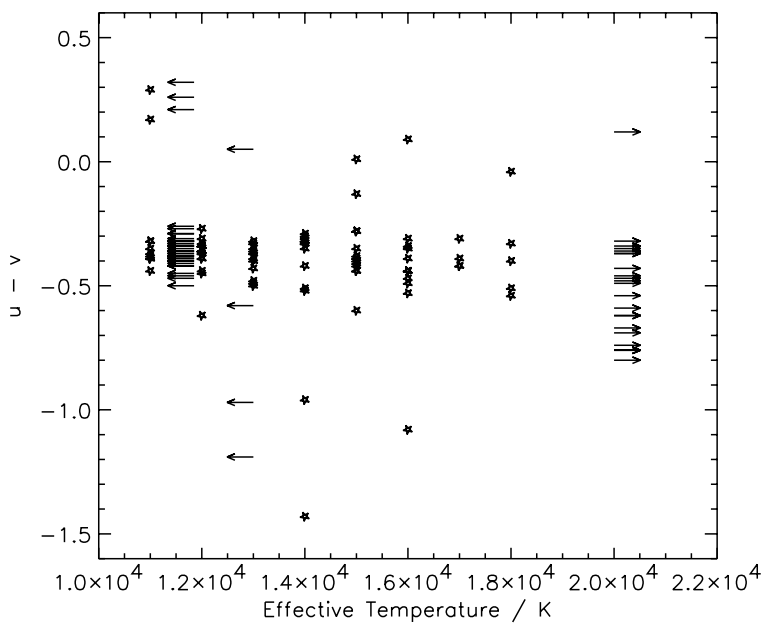

Fig. 4. Photographic colours plotted against estimated effective temperatures. For some of the stars with upper limits for the effective temperature, the symbols have been slightly shifted to improve the clarity.

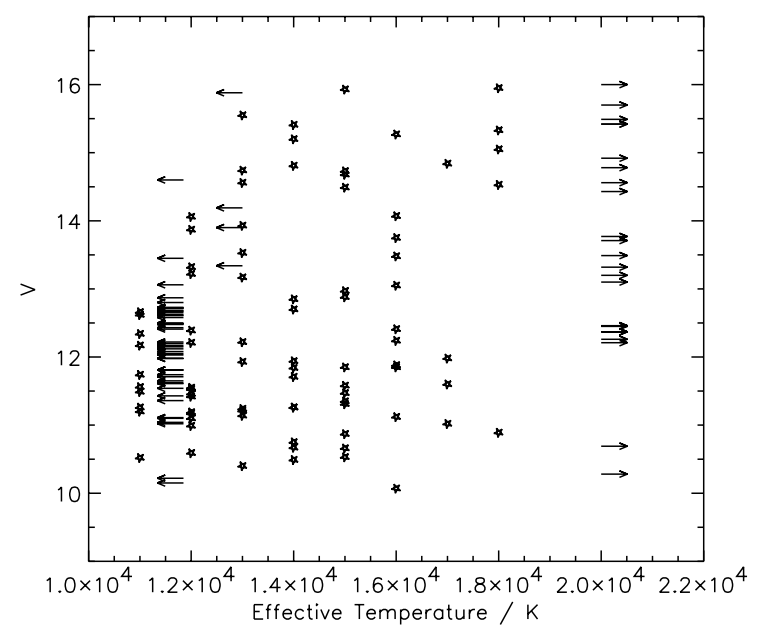

Fig. 5. Photographic $v$ magnitudes plotted against estimated effective temperatures. For some of the stars with upper limits for the effective temperature, the symbols have been slightly shifted to improve the clarity.

In Fig. 4, the effective temperature estimates are plotted against the photographic colour and no significant correlation is found. As discussed by DSH, this probably reflects a combination of two factors, viz. intrinsic errors in our colour determinations (that were estimated in Sect. 2.1 to be of the order of 0.2 magnitudes) and spatial variations in the reddening. However a consequence of this lack of correlation (and the blending problems discussed in Sect 2.1) is that we cannot be certain that our sample of early-type objects is even near to being complete.

The apparent $V$ photographic magnitudes are plotted against the effective temperature estimates in Fig. 5. Both the hot and cool targets seem to have a similar distribution in magnitudes, with tentative evidence for the hotter stars being marginally fainter. At first sight, this is surprising as the hotter stars should be intrinsically more luminous. However they will also have a lower 


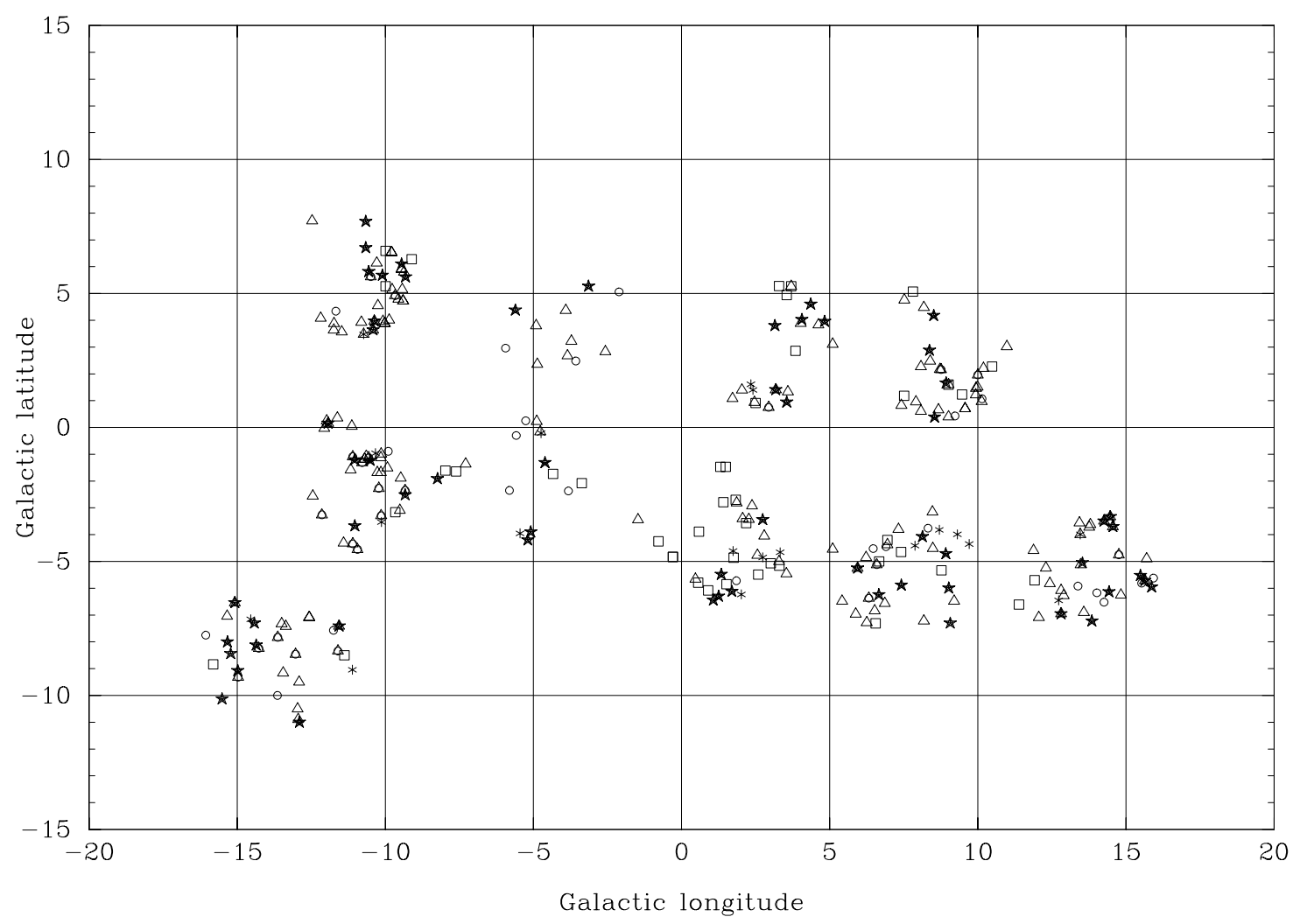

Fig. 6. The Galactic longitude and latitude coordinates of the stars in the 10 fields with the symbols representing different spectral classifications. As described in Sect.3, the classifications are early-B type (solid stars), mid B-type (open circles), late-B and AB-types (open triangles), late-types (open squares) and PN (asterisks). The observed distributions are not reliable indicators of the true number density of each spectral type (due to the incompleteness arguments given in Sect. 2). However it is clear that the early-B types in general lie several degrees from the plane, indicating that they may be runaways or evolved PAGB stars (see Sect. 4 for details). The relative paucity of objects at $l \sim-5$ (fields 454 and 393) are indicative of the poor observing conditions during which the spectra were taken and hence the number of stars with reliable spectral types are considerably lower than in the other fields.

space density, which could explain the observed distribution. Alternatively, there might be a significant number of sub-luminous objects in our hot star sample. To distinguish such objects would require far higher signal-to-noise and spectral resolution than presented here.

DSH presented illustrative estimations of distances to the early-type stars in the initial sample in three fields. In particular, they arbitrarily assumed a value of $E(B-V) \simeq$ 0.3 , similar to that found for LS4825 - a star that is believed to be beyond the Galactic Centre (Ryans et al. 1997). Adopting the same value for the reddening, our early-B-type targets (which lie in a magnitude range of $12.0 \lesssim V \lesssim 16.0$ ) would map onto a distance scale of 4 to $30 \mathrm{kpc}$. As discussed by DSH, the extinction towards these targets will be highly variable and this will lead to major uncertainties in these distances. For this reason, we have not attempted to estimate distances to individual stars and emphasize that the distance range is purely illustrative and consistent with some of the objects being close to the galactic centre. The nature and position of these targets will require both reliable estimates of the extinction (from CCD photometry) and of the atmospheric parameters (from high dispersion spectroscopy)
In Fig. 6 we show the Galactic longitude and latitude of the early-type targets in comparison with those of other types. The latitudes of the early-types are generally in the region $2^{\circ} \lesssim|b| \lesssim 10^{\circ}$, with a few stars lying closer to the plane. The distribution of stars is not representative of the true surface density of each type of object due to the incompleteness of our survey (as discussed in Sect. 2), however it is useful to examine where our detected early-B types tend to lie in respect to the other objects and the Galactic plane. Obviously the field centres chosen for the plates will also affect the observed distribution (see Fig. 1). No obvious pattern of spatial distribution emerges for the early-B types, but it is clear that many of them do lie away from the plane with $|b| \gtrsim 2^{\circ}$. These are at similar distances from the plane as the stars analysed in detail by Smartt et al. (2001b, 1997) and Venn et al. (1998). The former two studies show that normal, massive B-type stars can exist in these regions, probably having been ejected from the plane soon after formation. However some of the targets are probably old, evolved, post-AGB or post-BHB stars with effective temperatures similar to those of the normal young stars. A more detailed discussion of the possible scenarios has been given in DSH. 
In conclusion, we have discovered a significant number of blue objects (56 in total) which have spectra indicative of early-type B stars lying towards the Galactic centre. An estimation of atmospheric parameters indicates that 32 of these have effective temperatures greater than or equal to $17000 \mathrm{~K}$. They have similar Galactic latitudes to the normal stars found by Smartt et al. (2001b, 1997) and hence it is likely that some of them are inner Galaxy, young massive objects. The brightest, hottest candidates will be targeted in a future observing campaign involving high-resolution, high signal-to-noise spectroscopic measurements. This should provide a sample of young stars which were born within the inner $3-4 \mathrm{kpc}$, and whose photospheric abundances are representative of the interstellar medium in this region. This will supplement the sample of stars analysed Smartt et al. (2001b) to probe the chemistry of the inner regions of the Galactic disk.

Acknowledgements. We wish to thank the staff of the AngloAustralian Observatory and UKSTU at the Royal Observatory Edinburgh for obtaining additional Schmidt plates and for assistance with the low dispersion spectroscopy. In particular it is a pleasure to thank Paul Cass and Malcolm Hartley of the AAO for their expert and helpful assistance with the operation of the UKST and the often difficult fibering up process. We are grateful to Harvey MacGillivray and Eve Thomson for measuring the plates on SuperCOSMOS. Part of the data reduction was undertaken on the PPARC funded Starlink node at Queen's University. PPARC financial support for SJS and the visiting fellowship programme at QUB is gratefully acknowledged.

\section{References}

Bresolin, F., Kudritzki, R. P., Mendez, R. H., \& Przybilla, N. 2001, ApJ, in press [astro-ph/0012372]

Conlon, E. S., Dufton, P. L., Keenan, F. P., \& McCausland, R. J. H. 1993, ApJ, 408, 593

Claret, A. 1995, A\&AS, 109, 441

Drinkwater, M., \& Holman, B. 1996, Flair Data Reduction with IRAF, AAO Internal publications

Dufton, P. L., Smartt, S. J., \& Hambly, N. C. 1999, A\&AS, 139,231

Gummersbach, C. A., Kaufer, A., Schäfer, D. R., Szeifert, T., \& Wolf, B. 1998, A\&A, 342, 426
Hambly, N. C., Miller, L., MacGillivray, H. T., Herd, J. T., \& Cormack, W. A. 1998, MNRAS, 298, 897

Hibbins, R. E., Dufton, P. L., Smartt, S. J., \& Rolleston, W. R. J. 1998, A\&A, 332, 681

Howarth, I. D., Murray, J., \& Mills, D. 1993, Starlink User Note 50.14

Howarth, I. D. 1998, Proceedings of the BMW-II conference, ed. I. D. Howarth, ASP Conf. Ser., 169

Korn, A. J., Becker, S. R., Gummersbach, C. A., \& Wolf, B. 2000, A\&A, 353, 655

Kurucz, R. L. 1991, Precision Photometry: Astrophysics of the Galaxy, ed. Philip, Upgren \& Janes (L. Davis Press, Schenectady)

McCarthy, J. K., Lennon, D. J., Venn, K. A., et al. 1995, ApJL, 455, 135

Monteverde, M. I., Herrero, A., Lennon, D. J., \& Kudritzki, R. P. 1997, ApJL, 474, 107

Parker, Q. A., \& Watson, F. G. 1994, IAU Symp., No. 161, 85

Reed, B. C. 1993, ApJS, 87, 367

Reed, B. C., \& Beatty, A. E. 1995, ApJS, 97, 189

Rolleston, W. R. J., Brown, P. J. F., Dufton, P. L., \& Howarth, I. D. 1996, A\&A, 315, 95

Rolleston, W. R. J., Dufton, P. L., McErlean, N. D., \& Venn, K. A. 1999, A\&A, 347, 728

Rolleston, W. R. J., Smartt, S. J., Dufton, P. L., \& Ryans, R. S. I. 2000, A\&A, 363, 537

Ryans, R. S. I., Dufton, P. L., Keenan, F. P., et al. 1997, ApJ, 490, 267

Smartt, S. J., \& Rolleston, W. R. J. 1997, ApJL, 481, 47

Smartt, S. J., Dufton, P. L., \& Rolleston, W. R. J. 1996a, A\&AS, 116, 483

Smartt, S. J., Dufton, P. L., \& Rolleston, W. R. J. 1996b, A\&A, 310,123

Smartt, S. J., Dufton, P. L., \& Lennon, D. J. 1997, A\&A, 326, 763

Smartt, S. J., Crowther, P. A., Dufton, P. L., et al. 2001a, in press

Smartt, S. J., Venn, K. A., Lennon, D. J., et al. 2001b, A\&A, 367,86

Tritton, S. B. 1983, United Kingdom Schmidt Telescope Handbook, Royal Observatory Edinburgh

Valdes, F. 1992, Guide to Multifiber Reduction Task DOFIBERS, NOAO

Venn, K. A., Smartt, S. J., Lennon, D. J., \& Dufton, P. L. 1998, A\&A, 334, 987 